\title{
36. GEOCHEMISTRY OF LABILE ORGANIC MATTER IN SEDIMENTS AND INTERSTITIAL WATER RECOVERED FROM SITES 651 AND 653, ODP LEG 107 IN THE TYRRHENIAN SEA ${ }^{1}$
}

\author{
R. Seifert, ${ }^{2}$ K.-C. Emeis, ${ }^{3}$ A. Spitzy, ${ }^{2}$ K. Strahlendorff, ${ }^{2}$ W. Michaelis,${ }^{2}$ and E.T. Degens ${ }^{2}$
}

\begin{abstract}
Sediment and interstitial water from Sites 651 and 653 (ODP Leg 107) were investigated by organic geochemical methods to characterize labile organic compound classes (amino compounds and carbohydrates) and to evaluate their progressive diagenetic and thermal degradation in deep-sea sediments.

Downhole distribution of dissolved organic carbon (DOC) appears related to redox zones associated with bacterial activity and of diagenetic recrystallization of biogenic tests and not so much to organic matter concentrations in ambient sediments. DOC ranges from 250 to $8300 \mu \mathrm{mol} / \mathrm{L}$ (3-100.1 ppm). Amino acids contribute $10 \%-0.3 \%$ of DOC; carbohydrates range from 78 to $5 \mu \mathrm{mol} / \mathrm{L}$.

Rate of degradation of amino acids by thermal effects and/or bacterial activity at both sites (significantly different in sedimentation rates: average $41 \mathrm{~cm} / 1000 \mathrm{yr}$ in the top $300 \mathrm{~m}$ at Site 651 , average $3.9 \mathrm{~cm} / 1000 \mathrm{yr}$ in the Pliocene/Quaternary sequence at Site 653 to $220 \mathrm{mbsf}$ ) is more dependent on exposure time rather than on the depth within the sediment column. Variability in neutral, acidic, and basic amino acid fractions of total amino acids (with a range of 1.1$0.02 \mu \mathrm{mol} / \mathrm{g}$ sediment; up to $2.5 \%$ of organic carbon) varies with carbonate content and by differences in thermal stability of amino acids.

Distribution patterns of monosaccharides are interpreted to result from differences in organic matter sources, sedimentation rates, and the degree of organic matter decomposition prior to and subsequent to burial. Total particulate carbohydrates range from 1.82 to $0.21 \mu \mathrm{mol} / \mathrm{g}$ sediment and contribute about $8 \%$ to the sedimentary organic matter. Investigation of trace metals in the interstitial waters did not show any correlation of either DOC, amino compounds, or carbohydrates.
\end{abstract}

\section{INTRODUCTION AND GEOLOGICAL BACKGROUND}

Leg 107 of the Ocean Drilling Program drilled a transect of seven sites across the Tyrrhenian sub-basin of the Mediterranean Sea (Fig. 1). The transect was designed to (1) clarify the age of back-arc spreading behind the subduction zone of the Calabrian arc by recovering basaltic basement rocks in the Marsili and Vavilov basins; and (2) to study the association of back-arc evolution with the timing and mechanism of passive margin evolution on the eastern continental margin of Sardinia.

Results of drilling on Leg 107 document the history of tectonic movement and the evolution that led to the present configuration of the back arc and the passive margin (Kastens, Mascle, et al., 1987). Aspects of regional geology and Mediterranean geological history with respect to the late Miocene salinity crisis are discussed in numerous articles in the present volume.

Of particular interest among the sites drilled during Leg 107 are Site 651 in the Vavilov Basin, which is floored by basalt, and Site 653 on the Sardinian margin. At the former site, chemically altered sediments were recovered at the contact with a complex basement unit. Strata from Holes 653A and 653B include a largely undisturbed and complete pelagic section of Messinian to Pleistocene age.

We extended the scope of shipboard analyses presented in the site chapters (Kastens, Mascle, et al., 1987) by studying samples

\footnotetext{
${ }^{1}$ Kastens, K.A., Mascle, J., et al., 1990. Proc. ODP, Sci. Results, 107: College Station, TX (Ocean Drilling Program).

2 Geologisch-Paläontologisches Institut, Universităt Hamburg, D-2000 Hamburg 13, F.R.G. U.S.A
}

from these two sites in greater detail. In addition to interstitial water properties determined onboard the JOIDES Resolution, we measured organic constituents (organic carbon, carbohydrates, amino compounds) in pore waters and in the respective squeezed sediment cakes. Trace metals were determined in the pore waters of Holes $651 \mathrm{~A}$ and $653 \mathrm{~A}$ and $\mathrm{B}$ in order to evaluate (1) possible interactions of dissolved organic and inorganic constituents, and (2) possible hydrothermal influence on pore water composition at Site 651 .

The investigation of interstitial water chemistry in relation to the composition of the pelagic sediments in the Tyrrhenian Sea had two objectives. The first objective was to trace the composition and quantity of labile organic constituents through successive stages of organic matter remineralization and sediment diagenesis. Profiles of dissolved organic matter concentrations and compositional differences are useful in following bacterial or other diagenetic processes that convert particulate to dissolved organic matter. Recrystallization of biogenic hard parts of microorganisms, for example, should result in different compositions relative to bacterial degradation of organic matter.

The second area of investigation concerns possible hydrothermal influences on the composition of interstitial waters and the range of organic constituents in sediment and pore water. Sediment overlying young basaltic ocean floor at Site 651 , where there is a high geothermal gradient of about $150^{\circ} \mathrm{C} / \mathrm{km}$, would be expected to show thermal effects on organic matter, whereas sediment from Site 653 (geothermal gradient approximately $80^{\circ} \mathrm{C} / \mathrm{km}$ ) should show predominantly biogenic effects. An investigation of carbohydrates and amino compounds in the pore water and in the sediment would resolve differences of thermal history and input characteristics for the two sites.

A brief description of the sedimentological facies and sediment type for each of the two sites has been excerpted from Kastens, Mascle, et al. (1987) as background for our geochemical studies. 


\section{R. SEIFERT ET AL.}

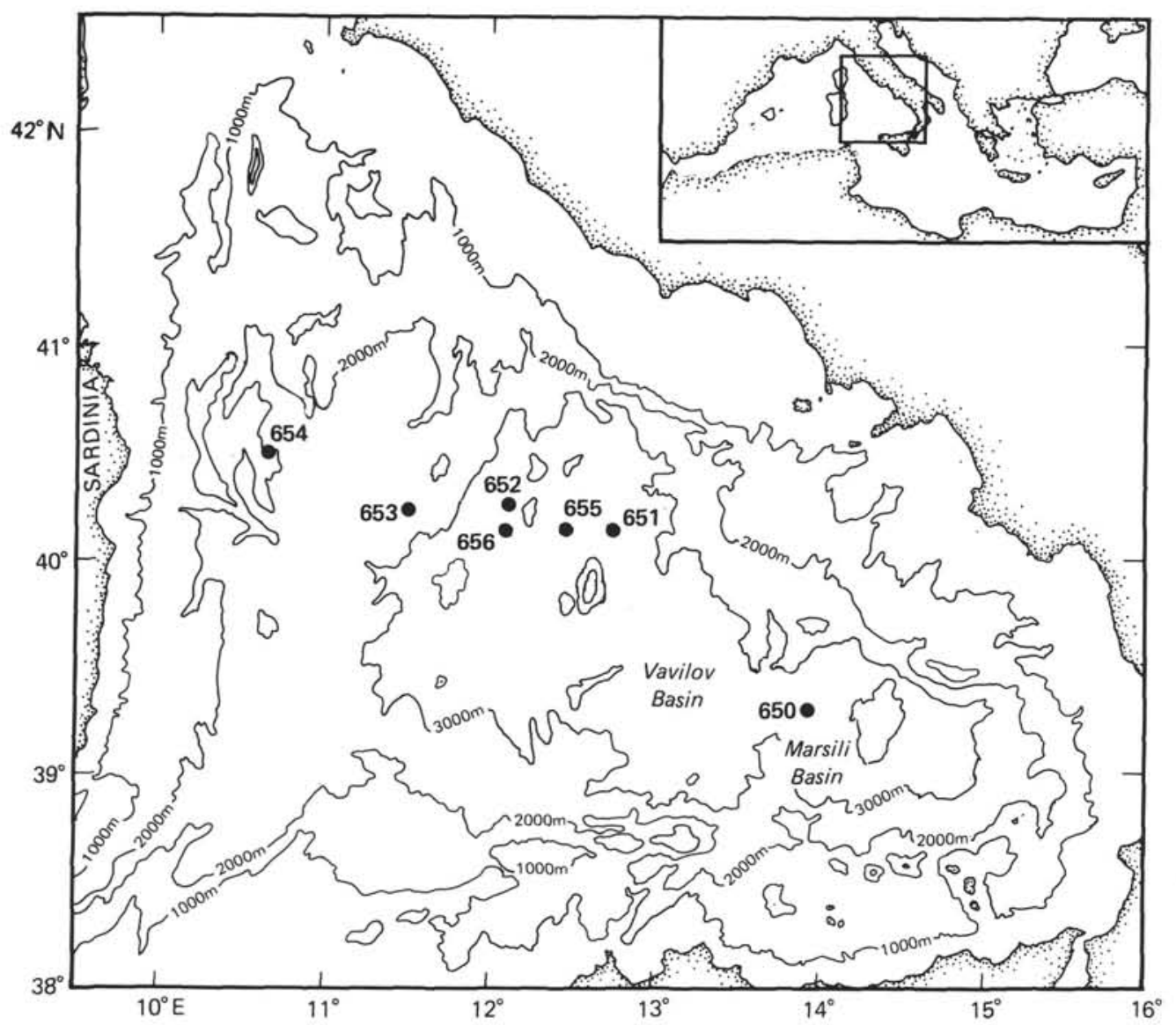

Figure 1. Location map of sites drilled during ODP Leg 107.

\section{Site 651}

Site 651 is located on the abyssal plain of Vavilov Basin in $3578 \mathrm{~m}$ water. The basaltic oceanic crust here is late Pliocene in age, and heat flow measurements showed a high gradient of $14.6^{\circ} \mathrm{C} / 100 \mathrm{~m}$. Two sedimentary units and a basement unit were recognized at Site 651: sedimentary Unit I ( $0-136 \mathrm{mbsf})$ is comprised of volcanogenic sediment with variable contributions of marly nannofossil-rich mud. The unit is of late Pleistocene age, and the sediments accumulated at rates of about $70 \mathrm{~cm} / 1000 \mathrm{yr}$. Average $\mathrm{CaCO}_{3}$ content is $17.7 \% \pm 8.2 \%$ and ranges from $1.9 \%$ to $42.2 \%$.

Sedimentary Unit II (136-387.6 mbsf) consists of marly nannofossil chalks and oozes, volcanic ash, calcareous siltstone, mudstones, and claystones. Dolostones and metalliferous claystones and dolostones (Mn-Fe-enriched) are prominent in Subunit IIb (347.8-387.6 mbsf) at the contact of Unit II with the basaltic basement. In the upper part of Unit II, 11 individual organic-carbon-rich sapropels and sapropelic layers were found in Cores 651A-18R, $-27 R,-28 R$, and $-34 R$ to $-37 R$. The maximum value for $C_{\text {org }}$ of $4.16 \%$ by weight was encountered in Sample 651A-36R-1, 54-56 cm. Carbonate content in Subunit IIa averages $21.7 \% \pm 17.7 \% \mathrm{CaCO}_{3}$ and ranges from $0.2 \%$ to $69.6 \%$. In Subunit IIb, in the altered and in places brightly colored dolostones, $\mathrm{CaCO}_{3}$ averages $47.2 \% \pm 13 \%$ and ranges from $12.4 \%$ to $64.9 \%$. Basement is comprised of a complex assemblage of serpentinized peridotite, basalts, basaltic breccia, dolerites, metasediments, and metadolerites.

\section{Site 653}

Site 653 was drilled close to DSDP Site 132 on the eastern rim of the Cornaglia Basin in $2817 \mathrm{~m}$ water depth. The geother- mal gradient at this site decreases with depth and has a mean value of $80^{\circ} \mathrm{C} / \mathrm{km}$ in the top $200 \mathrm{~m}$. Two major sedimentary Units were recognized in the $264.3 \mathrm{~m}$ penetrated. Unit I (0-220 mbsf in Hole 653A and 0-216 mbsf in Hole 653B) was deposited in an open marine hemipelagic to pelagic sedimentary environment. Sediments are composed of gray and brown nannofossil oozes, foraminiferal nannofossil oozes and minor mud, which accumulated at an average rate of $4 \mathrm{~cm} / 1000 \mathrm{yr}$ over the Pliocene/Pleistocene. Carbonate content varies widely due to the abundance of clastic sand and muds, as well as volcaniclastic constituents. Average $\mathrm{CaCO}_{3}$ in Subunit Ia of Hole 653B (0-86 mbsf) is $39.7 \% \pm 10.3 \%$, while Subunit Ib (86-209.1 mbsf) averages $60.6 \pm 5.7 \% \mathrm{CaCO}_{3}$ with less clastic dilution. Fourteen discrete sapropel and sapropelic intervals were encountered in Subunit Ia of Hole 653B while they appear to be absent in Subunit $\mathrm{Ib}$. The highest number of sapropels was found in Cores $653 \mathrm{~B}-7 \mathrm{H}$ and $-8 \mathrm{H}$ between 55 and 65 mbsf (Emeis et al., this volume). The maximum concentration of organic carbon of 4.2 wt $\%$ was encountered in Sample 653B-7H-4, 29-31 cm. Subunit Ic marks the transition from subaerial deposits of Messinian sediments in sedimentary Unit II to the marine sediments of Unit I and consists of calcareous marine sediments (nannofossil oozes and marls), which are colored red and yellow by migrating iron from the underlying lithologic Unit II (220-240.7 mbsf in Hole 653A and 216-264 mbsf in Hole 653B). Average $\mathrm{CaCO}_{3}$ content in Subunit Ic is $52.2 \% \pm 7.2 \%$ with a relatively narrow range of $41.9 \%-60.4 \%$.

Unit II represents sedimentation in a restricted marine evaporitic and subaerial environment during the Messinian desiccation of the Tyrrhenian Sea. Dark gray gypsum-bearing sand, laminated gypsum, and dark gray to olive gray and red dolomitic nannofossil mud with lenticular gypsum characterize the 
upper Messinian section. Occurrence of brightly colored red and yellow bands of iron-rich precipitates increases downsection. Carbonate content averages $15.7 \% \pm 7.1 \% \mathrm{CaCO}_{3}$ and ranges from $8.1 \%$ to $24.1 \%$. Correlation of Messinian sediment within the few meters distance of Holes 653A and 653B proved to be difficult, a fact which suggests considerable lateral facies variabiltiy within the Messinian depositional environment.

\section{SAMPLES AND METHODS}

Samples investigated here are listed in Tables 1 and 2 along with a brief description of sediment facies. Samples of interstitial waters were obtained by standard shipboard techniques (Gieskes and Peretsman, 1986). Two 5-mL aliquots of each sample were sealed in pre-combusted glass ampoules and immediately frozen. Trace elements were determined by graphite furnace atomic absorption spectroscopy on one sample aliquot.

Dissolved organic carbon in the interstitial waters was determined by an instrument that employs UV oxidation coupled to an infrared detector for detection of $\mathrm{CO}_{2}$. The sample was acidified and purged prior to DOC oxidation to expel inorganic $\mathrm{CO}_{2}$ (Mueller and Bandaranayake, 1983). Dissolved amino compounds were measured following hydrolysis of 1-3 mL sample in $6 \mathrm{~N}$ $\mathrm{HCl}$ under argon for $23 \mathrm{hr}$ at $110^{\circ} \mathrm{C}$. Analyses were performed on a Biotronic Amino Acid Analyzer and quantified against standards. Particulate amino compounds in squeezed cakes were measured after hydrolysis of 50-150 mg dried and crushed subsamples under the same conditions.

Concentrations of carbohydrate monomers were measured after acid hydrolysis of $50-100 \mathrm{mg}$ freeze-dried and ground sediment or 1-3 mL interstitial water. Hydrolysis was carried out with $2 \mathrm{~N} \mathrm{HCl}$ at $100^{\circ} \mathrm{C}$ for $3.5 \mathrm{hr}$ under argon atmosphere in precombusted glass ampoules. The samples were analyzed and compared to standards on a Biotronik Sugar Analyzer by liquid chromatography (Mopper, 1977; Mopper et al., 1978) after hydrolysates had been desalted by electrodialysis. Details of all organic geochemical methods are given in Michaelis and Ittekkot (1982). At present, we have no data to decide whether artifacts are introduced during sampling and interstitial water squeezing in the case of the dissolved fractions. Comparison of in-situ filtrated water and water obtained from squeezing (Seifert et al., unpubl. data) suggests that the squeezing method is advantageous because of frequent seawater dilution in the in-situ sam- ples. Considering the rather dramatic changes in pressure, temperature, and oxygen content of the environment, we cannot exclude the possibility that DOC changes considerably in quality and quantity. Differences in the samples are obvious, however, and possible sampling artifacts are presumed to be significantly less important than differences in in-situ conditions.

\section{RESULTS AND DISCUSSION}

\section{Dissolved Organic Carbon}

DOC values from 367 to $3,292 \mu \mathrm{mol} / \mathrm{L}$ were detected in pore waters from Hole 651A (Table 3), whereas the values for Holes $653 \mathrm{~A}$ and $653 \mathrm{~B}$ are in the range of $258-8,340 \mu \mathrm{mol} / \mathrm{L}$ (Table 4). The range of DOC concentrations agrees reasonably well with results from other deep-sea and nearshore sediments, although the results appear to be on the low side due to low sedimentary organic carbon concentrations in the pelagic and hemipelagic sediments of the Tyrrhenian Sea (e.g., Emeis and Mycke, this volume). For example, Michaelis et al. (1982) reported concentrations from 2,580 to $17,000 \mu \mathrm{mol} / \mathrm{L}$ from organic-rich sediments of the Gulf of Mexico; Emeis et al. (1987) found values from 4,000 to $12,000 \mu \mathrm{mol} / \mathrm{L} \mathrm{DOC}$ from interstitial waters of the northwest Atlantic Ocean. Interstitial waters from nearshore sediment of the Atlantic Ocean investigated by Henrichs and Farrington (1987) ranged from 1,000 to $9,160 \mu \mathrm{mol} / \mathrm{L}$ DOC.

Profiles of DOC concentrations show distinct downhole changes (Fig. 2) which cannot be attributed to obvious lithological differences. In both sections organic-rich sapropel layers were found, which are concentrated between 55 and $65 \mathrm{mbsf}$ in Hole 653A, and between 310 and 350 mbsf in Hole 651A. None of these potential sources of dissolved organic matter appear to influence the distribution of DOC, however, and thus the observed variability in DOC concentrations with depth may result from DOC production or loss that is related more to diagenetic changes than to abundance of sedimentary organic matter and lithology.

One of the diagenetic zones that control DOC production or loss is, e.g., related to decrease in interstitial sulfate, which coincides with DOC minima at both sites (i.e., Sample 651A-7R-2, $140-150 \mathrm{~cm}$; 653B-7H-5, 140-150 cm; Table 3, 4). This coincidence may result from high microbial activity and consumption of dissolved organic matter in this zone. Also the high amino

Table 1. Samples and lithofacies of Hole 651A.

\begin{tabular}{lrlrl}
\hline \multicolumn{1}{c}{ Sample } & $\begin{array}{r}\text { Depth } \\
\text { (mbsf) }\end{array}$ & \multicolumn{1}{c}{ Description } & $\mathrm{CaCO}_{3}$ & $\mathrm{C}_{\text {org }}$ \\
\hline 1R-3, 140-150 & 4.4 & Calcareous-rich silty mud & 22.7 & n.d. \\
3R-1, 54-67 & 10.8 & Calcareous mud & 27.2 & n.d. \\
4R-3, 54-67 & 23.3 & Gray clayey nanno-rich mud & 18.5 & n.d. \\
7R-2, 140-150 & 52.4 & Pumice & 1.9 & 0.00 \\
12R-1, 50-55 & 97.9 & Clayey-bearing carbonaceous mudstone & 42.2 & 0.00 \\
14R-3, 25-29 & 120.0 & Ash-bearing marly calcareous ooze & 19.7 & n.d. \\
17R-1, 25-28 & 146.0 & Silty mud & 21.0 & n.d. \\
18R-5, 140-150 & 162.3 & Marly nannofossil chalk & 34.1 & 1.30 \\
23R-1, 140-150 & 204.6 & Clayey mudstone & 6.1 & n.d. \\
26R-1, 140-150 & 233.7 & Clayey mudstone & 4.4 & 0.11 \\
34R-1, 140-150 & $310.7^{*}$ & Marly nannofossil chalk & 33.9 & 0.22 \\
37R-2, 20-27 & 339.9 & Calcareous claystone & 47.0 & n.d. \\
37R-4, 20-27 & $342.9^{*}$ & Foraminifer-nannofossil chalk & 34.4 & n.d. \\
39R-2, 68-74 & 359.7 & Metalliferous dolostone & 45.1 & n.d. \\
39R-3, 127-133 & $361.8^{*}$ & Metalliferous dolostone & 45.1 & n.d. \\
39R-4, 69-75 & 362.7 & Metalliferous dolostone & 49.9 & n.d. \\
40R-2, 55-60 & 369.3 & Metalliferous dolostone & 49.3 & 0.00 \\
40R-3, 39-44 & 370.6 & Metalliferous dolostone & n.d. & n.d. \\
40R-4, 55-60 & 372.3 & Metalliferous dolostone & 50.4 & n.d. \\
41R-2, 14-20 & 378.4 & Dolostone & 13.0 & n.d \\
41R-4, 14-20 & 381.4 & Dolostone & 60.9 & 0.0 \\
\hline
\end{tabular}

* = Near sapropels; n.d. $=$ not determined because high inorganic carbon content resulted in error with calculating organic carbon by difference. 
Table 2. Samples and lithofacies of Holes 653A and 653B.

\begin{tabular}{lrllll}
\hline \multicolumn{1}{c}{ Sample } & $\begin{array}{r}\text { Depth } \\
\text { (mbsf) }\end{array}$ & \multicolumn{1}{c}{ Description } & $\mathrm{CaCO}_{3}$ & $\mathrm{C}_{\text {org }}$ \\
\hline A 1H-2, 140-150 & 2.9 & Nannofossil ooze & 36.6 & n.d. \\
B 1H-4, 39-46 & 4.9 & Marly nannofossil ooze & 23.5 & 1.12 \\
B 2H-5, 131-137 & 13.5 & Nannofossil ooze & 65.2 & 0.13 \\
B 3H-4, 143-149 & 22.0 & Nannofossil ooze & 53.8 & 0.17 \\
B 4H-3, 142-149 & 31.5 & Foraminifer-nannofossil ooze & 20.9 & 0.26 \\
B 5H-5, 142-148 & $44.0^{*}$ & Foraminifer-nannofossil ooze & 49.3 & 0.61 \\
A 6H-5, 140-150 & $49.0^{*}$ & Marly nannofossil ooze & 38.7 & n.d. \\
B 6H-4, 140-148 & $51.9^{*}$ & Foraminifer-nannofossil ooze & 59.0 & 0.00 \\
B 6H-5, 58-66 & $52.6^{*}$ & Foraminifer-nannofossil ooze & 58.7 & 0.57 \\
B 7H-3, 143-149 & $59.4^{*}$ & Marly foram-nannofossil ooze & 46.8 & 0.57 \\
B 7H-5, 140-150 & $62.8^{*}$ & Foraminifer-nannofossil ooze & 36.3 & 0.25 \\
& & with ash and sapropel layer & & \\
B 8H-2, 50-56 & 66.9 & Foraminifer-nannofossil ooze & 38.4 & 0.65 \\
B 9X-2, 120-126 & 77.1 & Marly foram-nannofossil ooze & 55.1 & 0.69 \\
A11H-5, 140-150 & 96.4 & Foraminifer-rich nannofossil ooze & 56.4 & n.d. \\
B12X-3, 142-148 & 107.0 & Foraminifer-rich nannofossil ooze & 67.3 & 0.00 \\
B13X-4, 142-148 & 118.0 & Foraminifer-nannofossil ooze & 60.1 & n.d. \\
B14X-2, 104-110 & 124.1 & Foraminifer nannofossil ooze & 54.3 & n.d. \\
B16X-3, 140-148 & 144.9 & Foraminifer nannofossil ooze & 57.1 & 0.03 \\
B17X-3, 140-148 & 154.4 & Foraminifer-nannofossil ooze & 57.7 & 0.15 \\
B18X-4, 142-150 & 165.5 & Foraminifer-nannofossil ooze & 61.0 & 0.29 \\
B19X-4, 115-121 & 174.1 & Foraminifer-nannofossil ooze & 59.2 & n.d. \\
B20X-3, 141-148 & 182.4 & Foraminifer-nannofossil ooze & 56.2 & n.d. \\
B21X-3, 141-148 & 191.4 & Foraminifer-nannofossil ooze & 68.4 & n.d. \\
B22X-3, 80-86 & 201.0 & Foraminifer-nannofossil ooze & 56.1 & n.d. \\
B23X-3, 80-86 & 210.5 & Foraminifer-nannofossil ooze & 46.8 & n.d. \\
\hline & & & & \\
\hline
\end{tabular}

$\mathrm{A}=$ Hole $653 \mathrm{~A}, \mathrm{~B}=$ Hole $653 \mathrm{~B} ;{ }^{*}=$ Near sapropels; $\mathrm{n} . \mathrm{d} .=$ not determined because high inorganic carbon content resulted in error with calculating organic carbon by difference.

acid content in the interstitial water is accompanied by high $\beta$ alanine ( $\beta$-Ala) and $\gamma$-amino- butyric acid $(\gamma$-ABA) percentages in the amino acid fraction of the sediment in Hole $653 \mathrm{~A}$; the implications of these observations will be discussed below.

\section{Amino compounds}

Amino acids in pore waters range from 1.41 to $13.5 \mu \mathrm{mol} / \mathrm{L}$, which amounts to $9.6 \%$ and $0.3 \%$, respectively, of the DOC (Tables 5 and 6). Data for amino acids and amino sugars in sediment samples are listed for Sites 651 and 653 in Tables 7 and 8, respectively. The amino sugar contents varies from 0 to 145 $\mathrm{nmol} / \mathrm{g}$ at Site 651 and from 24.2 to $256 \mathrm{nmol} / \mathrm{g}$ at Site 653.

The vertical distributions of total hydrolyzable amino acids for Holes 651A and 653B are depicted in Figures 3 and 4. Both profiles show a general decrease with depth with only minor variability in the Pliocene samples. The neutral amino acids are dominant in samples of Hole 651A (Fig. 3). In the lower Pleis- tocene and upper Pliocene samples acidic amino acids are more abundant than are basic amino acids. In the predominantly dolomitic sediment at $\mathbf{3 5 7 . 5} \mathrm{mbsf}$, the percentage of basic amino acids increases compared to the acidic fraction. This increase could be attributable to the loss of acidic amino acids during recrystallization of biogenic carbonate tests, or to a lower contribution of basic amino acids, which are preferentially adsorbed on clay minerals, in the carbonate-rich dolomitic sediments. Because acidic amino acids are more stable under high temperature conditions than are basic amino acids in unaltered calcite shell materials (Vallentyne, 1969), the loss of acidic compounds must be preceeded by the liberation from recrystallization of hard parts prior to thermal degradation. Ornithine, a basic amino acid, together with neutral-amino acids glycine and alanine are highest in the samples from the dolomitized interval (Table 7). Ornithine is the most stable amino acid in studies on the thermal stability of amino components in humic acid (Khan and Sowden, 1971). Amino sugars (AS), which are the least thermally stable amino compound, are absent in the deepest part of Hole 651 (Table 7).

Of the neutral, acidic, and basic amino acids at Site 653 (Fig. 4), the neutral compounds show an increasing dominance with depth. In general, the acidic amino acids are more abundant than the basic amino acids in all samples below 20 mbsf. Exceptions are Samples 653B-8H-2 and 653B-4H-3 which are distinguished by low carbonate contents. Acidic amino acids are known to be major constituents of the organic matrix in calcified skeletons (Weiner et al., 1983; Degens, 1976; Schroeder, 1975; King and Hare, 1972) and are also easily adsorbed onto carbonate particles (Carter and Mitterer, 1978). On the other hand, they are less apt to be incorporated into the lattice of clay minerals than are basic amino acids (Hedges and Hare, 1987). Therefore it seems feasible that the early diagenetic decomposition of amino acids leads to a relative enrichment of acidic relative to basic amino acids in carbonate-rich sediments whereas the acidic amino acids decrease relative to basic amino acids in clay-rich sediments. Laboratory decomposition experiments under conditions of high pressure and temperature on Okhotsk Sea sediments support this suggestion (Maita et al., 1982).

Several studies on amino acids in core samples show a downhole increase of the nonprotein amino acids $\gamma$-amino butyric acid ( $\gamma$-ABA) and $\beta$-alanine ( $\beta$-Ala) relative to the total amino acid fraction (e.g., Whelan, 1977). Both components are common in sediment samples (Aizenshtat et al., 1973; Hare, 1973). $\gamma$-ABA and $\beta$-Ala are thought to be biological decarboxylation products of other amino acids such as glycine (Gly) and aspartic acid (Asp) (Aizenshtat et al., 1973; Vallentyne, 1964), whereas

Table 3. Results of interstitial water analyses, Hole $651 \mathrm{~A}$.

\begin{tabular}{|c|c|c|c|c|c|c|c|c|c|c|c|c|c|c|c|}
\hline Sample & $\begin{array}{l}\text { Depth } \\
\text { (mbsf) }\end{array}$ & $\mathrm{pH}$ & Alk & $\mathrm{Ca}^{2+}$ & $\mathrm{Mg}^{2+}$ & $\mathrm{SO}_{4}{ }^{2-}$ & $\mathrm{Cl}^{-}$ & Sal & $\mathrm{Ca} / \mathrm{Mg}$ & $\mathrm{SiO}_{2}$ & $\mathrm{~K}^{+}$ & $\mathrm{Sr}$ & Mn & $\mathrm{Fe}$ & DOC \\
\hline $1 \mathrm{R}-3,140-150$ & 4.4 & 7.54 & 3.59 & 11.46 & 59.90 & 28.21 & 663 & 39.5 & 0.19 & - & - & - & - & - & - \\
\hline $3 R-1,54-67$ & 10.8 & 7.84 & 5.01 & 9.76 & 26.69 & 13.64 & 574 & 37.0 & 0.37 & 668 & 26.5 & 156 & 16.0 & 0.9 & 766 \\
\hline $4 R-3,54-67$ & 23.3 & 7.78 & 6.34 & 18.25 & 40.34 & 20.55 & 493 & 40.0 & 0.45 & 367 & 27.3 & 184 & 0.1 & 6.6 & 808 \\
\hline $7 R-2,140-150$ & 52.4 & 8.19 & 2.49 & 2.94 & 25.18 & 13.12 & 612 & 39.0 & 0.12 & 189 & 17.1 & 114 & 17.0 & 0.5 & 367 \\
\hline $12 \mathrm{R}-1,50-55$ & 97.9 & - & - & 48.65 & 11.23 & 20.93 & 621 & 40.0 & 4.33 & - & - & - & - & - & - \\
\hline $14 \mathrm{R}-3,25-29$ & 120.0 & 7.09 & 1.47 & 30.67 & 13.48 & 23.98 & 584 & 39.5 & 3.76 & 257 & 21.7 & 579 & 57.6 & 5.2 & 1658 \\
\hline $18 \mathrm{R}-5,140-150$ & 162.3 & 7.59 & 1.99 & 23.33 & 22.48 & 18.62 & 594 & 39.0 & 1.05 & 473 & 18.9 & 563 & 21.0 & - & 1392 \\
\hline $23 \mathrm{R}-1,40-150$ & 204.6 & 7.65 & 1.68 & 24.64 & 15.78 & 19.74 & 559 & 39.0 & 1.56 & 689 & 17.1 & 346 & 36.5 & 0.6 & 1833 \\
\hline $26 \mathrm{R}-1,30-150$ & 233.7 & 7.48 & 2.07 & 19.49 & 41.39 & 28.81 & 582 & 39.2 & 0.47 & 333 & 13.2 & 188 & 41.2 & 0.8 & - \\
\hline $34 \mathrm{R}-1,140-150$ & 310.7 & 7.50 & 2.53 & 6.67 & 15.58 & 11.04 & 587 & 39.0 & 0.43 & 402 & 12.3 & 132 & 41.1 & 0.5 & 3292 \\
\hline $37 \mathrm{R}-4,20-27$ & 342.9 & - & - & 27.81 & 47.69 & 34.09 & 574 & 36.0 & 0.58 & 284 & 11.3 & 162 & 23.9 & 0.3 & 2450 \\
\hline $39 R-3,127-135$ & 361.8 & 7.50 & 2.03 & 30.73 & 50.55 & 31.78 & 576 & 39.0 & 0.61 & 387 & 9.9 & 113 & 7.3 & 0.4 & \\
\hline $39 R-4,69-75$ & 362.7 & - & - & 19.41 & 40.79 & 24.94 & 564 & 36.0 & 0.48 & - & - & - & - & - & - \\
\hline $40 \mathrm{R}-2,55-60$ & 369.3 & - & - & 19.66 & 41.39 & 25.76 & 522 & 32.5 & 0.47 & - & - & - & - & - & - \\
\hline $40 \mathrm{R}-3,39-44$ & 370.6 & - & - & 25.93 & 41.89 & 27.62 & 535 & 34.0 & 0.62 & - & - & - & - & - & - \\
\hline $41 \mathrm{R}-2,14-20$ & 378.4 & 7.59 & 2.03 & 13.11 & 39.89 & 26.35 & 509 & 32.0 & 0.33 & 177 & 8.6 & 95 & 7.1 & 3.3 & 2450 \\
\hline $41 R-4,14-20$ & 381.4 & - & - & 14.32 & 41.99 & 27.61 & 543 & 35.0 & 0.35 & - & - & - & - & - & - \\
\hline
\end{tabular}

Alkalinity, $\mathrm{Ca}^{2+}, \mathrm{Mg}^{2+}, \mathrm{SO}_{4}{ }^{2-}, \mathrm{Cl}^{-}, \mathrm{K}^{+}$in mmol/L; salinity in $\%$; $\mathrm{SiO}_{2}, \mathrm{Sr}, \mathrm{Mn}, \mathrm{Fe}$, and $\mathrm{DOC}$ in $\mu \mathrm{mol} / \mathrm{L}$. $-=$ not determined. 
Table 4. Interstitial water analyses, Site 653, Leg 107.

\begin{tabular}{|c|c|c|c|c|c|c|c|c|c|c|c|c|c|c|c|}
\hline Sample & Depth & $\mathrm{pH}$ & Alk & $\mathrm{Ca}^{2+}$ & $\mathrm{Mg}^{2+}$ & $\mathrm{SO}_{4}{ }^{2-}$ & $\mathrm{Cl}^{-}$ & Sal & $\mathrm{Ca} / \mathrm{Mg}$ & $\mathrm{SiO}_{2}$ & $\mathrm{~K}^{+}$ & $\mathrm{Sr}$ & $\mathrm{Mn}$ & $\mathrm{Fe}$ & DOC \\
\hline $1 \mathrm{H}-2,40-150$ & 2.9 & 7.68 & 2.99 & 6.9 & 51.1 & 24.6 & 606 & 37.5 & 0.13 & 363 & 14.6 & 110 & 61.2 & 0.5 & 350 \\
\hline $2 \mathrm{H}-5,140-150$ & 13.5 & 7.51 & 3.73 & 9.3 & 50.7 & 26.3 & 524 & 36.0 & 0.18 & - & - & - & - & - & \\
\hline $3 \mathrm{H}-4,143-149$ & 22.0 & - & - & 8.5 & 54.0 & 24.6 & 581 & 39.0 & 0.16 & 132 & 13.4 & 167 & 22.6 & 0.7 & 2700 \\
\hline $4 \mathrm{H}-3,42-149$ & 31.5 & - & - & 7.0 & 47.2 & 21.5 & 600 & 38.0 & 0.15 & 32 & 13.5 & 196 & 7.7 & 0.3 & 1383 \\
\hline $5 \mathrm{H}-5,142-148$ & 44.0 & - & - & 15.6 & 60.5 & 25.3 & 608 & 39.5 & 0.26 & 162 & 12.7 & 232 & 14.7 & 0.6 & - \\
\hline $6 \mathrm{H}-5,40-150$ & 49.0 & 7.49 & 2.80 & 18.4 & 66.5 & 30.5 & 550 & 38.0 & 0.28 & 323 & 12.9 & 219 & 20.3 & 3.0 & - \\
\hline $6 \mathrm{H}-4,140-148$ & 51.9 & - & - & 7.7 & 35.7 & 26.8 & 613 & 38.5 & 0.21 & 228 & 10.7 & 239 & 21.1 & 18.2 & - \\
\hline $7 \mathrm{H}-5,140-150$ & 62.8 & 7.38 & 3.25 & 15.3 & 59.7 & 17.6 & 623 & 40.0 & 0.26 & 425 & 12.5 & 260 & 28.6 & 4.3 & 258 \\
\hline $8 \mathrm{H}-2,50-56$ & 66.9 & - & - & 12.2 & 57.8 & 28.0 & 589 & 40.0 & 0.21 & 178 & 13.8 & 289 & 27.3 & 2.9 & 658 \\
\hline $11 \mathrm{H}-5,40-150$ & 96.4 & 7.11 & 2.66 & 18.9 & 53.3 & 24.0 & 634 & 40.0 & 0.36 & 294 & 13.3 & 338 & 27.1 & 6.6 & - \\
\hline $12 X-3,42-148$ & 107.0 & - & - & 21.1 & 63.6 & 34.2 & 665 & 42.0 & 0.33 & 217 & 13.4 & 378 & 12.7 & 0.5 & 1280 \\
\hline $13 X-4,142-149$ & 118.0 & - & - & 22.3 & 61.9 & 33.5 & 654 & 42.5 & 0.36 & 65 & 9.8 & 199 & 8.3 & 0.4 & 1542 \\
\hline $14 X-2,104-110$ & 124.1 & - & - & 21.8 & 59.7 & 34.1 & 630 & 44.5 & 0.37 & 226 & 9.6 & 403 & 13.7 & 0.9 & 8340 \\
\hline $16 \mathrm{X}-3,140-148$ & 144.9 & - & - & 26.9 & 61.9 & 34.5 & 621 & 45.0 & 0.43 & 259 & 12.9 & 415 & 13.5 & 20.0 & 6150 \\
\hline $17 X-3,140-148$ & 154.4 & - & - & 25.4 & 64.5 & 37.3 & 636 & 42.0 & 0.39 & 247 & 9.6 & 374 & 7.5 & 0.5 & 2800 \\
\hline $18 X-4,142-150$ & 165.5 & - & - & 28.6 & 67.5 & 40.5 & 683 & 44.0 & 0.42 & 98 & 9.9 & 396 & 13.6 & 1.6 & 2450 \\
\hline $21 X-3,141-148$ & 191.4 & - & - & 36.3 & 67.7 & 43.3 & 690 & 44.0 & 0.54 & 36 & 13.5 & 441 & 22.6 & 13.2 & 883 \\
\hline $22 X-3,80-86$ & 201.0 & - & - & 29.8 & 72.2 & 36.8 & 681 & 46.0 & 0.41 & 166 & 12.8 & 426 & 25.1 & 0.3 & - \\
\hline $23 X-3,80-86$ & 210.5 & - & - & 28.2 & 75.1 & 31.7 & 695 & 45.0 & 0.38 & 291 & 12.4 & 417 & 57.9 & 1.1 & 733 \\
\hline
\end{tabular}

Alkalinity, $\mathrm{Ca}^{2+}, \mathrm{Mg}^{2+}, \mathrm{SO}_{4}{ }^{2-}, \mathrm{Cl}^{-}, \mathrm{K}^{+}$, in mmol/L; salinity in $\%$; $\mathrm{SiO}_{2}, \mathrm{Sr}, \mathrm{Mn}, \mathrm{Fe}$, and $\mathrm{DOC}$ in $\mu \mathrm{mol} / \mathrm{L} ;-=$ not determined.
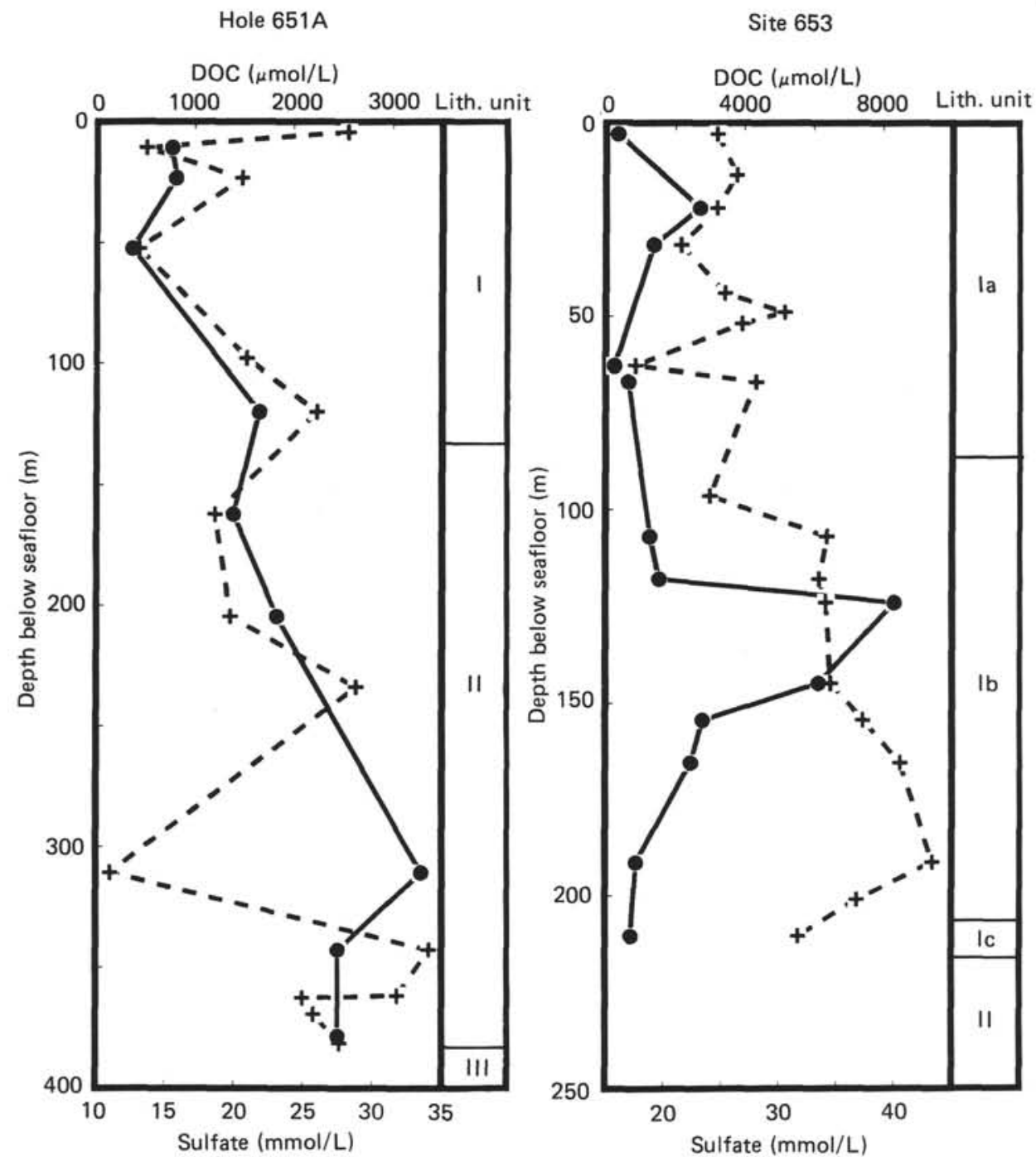

Figure 2. Downhole variations in dissolved organic carbon (DOC, solid lines) and sulfate (broken lines) at Hole $651 \mathrm{~A}$ and Site 653, respectively. Note the different depth scales. 
Table 5. Amino acids (AA) in interstitial waters, Hole $651 \mathrm{~A}$.

\begin{tabular}{|c|c|c|c|}
\hline Sample & $34 \mathrm{R}-1$ & $40 \mathrm{R}-2$ & $41 \mathrm{R}-2$ \\
\hline Asp & 5.2 & 8.9 & 5.2 \\
\hline Thr & 9.2 & 2.5 & 1.3 \\
\hline Ser & 6.7 & 12.8 & 5.8 \\
\hline Glu & 23.5 & 10.3 & 0.0 \\
\hline Gly & 16.9 & 25.3 & 10.6 \\
\hline Ala & 3.1 & 14.2 & 0.0 \\
\hline Val & 3.8 & 5.0 & 26.1 \\
\hline Ile & 2.6 & 1.5 & 2.4 \\
\hline Leu & 3.2 & 3.3 & 3.4 \\
\hline Tyr & 1.5 & 0.0 & 0.0 \\
\hline Phe & 1.7 & 0.0 & 1.4 \\
\hline$\beta \mathrm{Ala}$ & 2.8 & 0.0 & 3.8 \\
\hline$\gamma \mathrm{ABA}$ & 1.2 & 5.1 & 4.8 \\
\hline Orn & 8.0 & 7.7 & 24.0 \\
\hline Lys & 4.6 & 3.6 & 7.1 \\
\hline His & 4.4 & 0.0 & 1.7 \\
\hline Arg & 1.5 & 0.0 & 2.6 \\
\hline $\begin{array}{l}\text { Total AA } \\
(\mu \mathrm{mol} / \mathrm{L})\end{array}$ & 13.52 & 1.41 & 1.64 \\
\hline $\begin{array}{l}\text { Depth } \\
\text { (mbsf) }\end{array}$ & 310.7 & 369.3 & 381.4 \\
\hline $\begin{array}{l}\text { DOC } \\
(\mathrm{mg} / \mathrm{L})\end{array}$ & 39.5 & n.d. & 29.4 \\
\hline $\begin{array}{l}\text { AA/DOC } \\
(\%)\end{array}$ & 1.8 & n.d. & 0.31 \\
\hline
\end{tabular}

Table 6. Amino acids (AA) in interstitial waters, Site 653.

\begin{tabular}{lrrrrrrr}
\hline \multicolumn{1}{c}{ Hole } & $653 \mathrm{~A}$ & $653 \mathrm{~B}$ & $653 \mathrm{~B}$ & $653 \mathrm{~B}$ & $653 \mathrm{~B}$ & $653 \mathrm{~B}$ & $653 \mathrm{~B}$ \\
Sample & $1 \mathrm{H}-2$ & $7 \mathrm{H}-5$ & $8 \mathrm{H}-2$ & $12 \mathrm{X}-3$ & $16 \mathrm{X}-3$ & $17 \mathrm{X}-3$ & $22 \mathrm{X}-3$ \\
\hline Asp & 7.8 & 8.3 & 7.6 & 8.3 & 7.9 & 7.7 & 6.8 \\
Thr & 4.2 & 5.1 & 3.2 & 3.5 & 3.7 & 4.1 & 5.2 \\
Ser & 13.9 & 24.8 & 18.1 & 11.4 & 17.2 & 20.3 & 14.8 \\
Glu & 10.1 & 16.3 & 17.3 & 24.2 & 17.2 & 12.3 & 11.9 \\
Gly & 23.1 & 12.9 & 16.9 & 13.6 & 14.6 & 15.5 & 12.5 \\
Ala & 8.1 & 4.2 & 9.6 & 4.2 & 9.1 & 7.7 & 11.5 \\
Val & 3.8 & 2.3 & 3.7 & 4.4 & 4.8 & 3.2 & 3.7 \\
Ile & 2.2 & 2.6 & 2.3 & 2.7 & 2.4 & 2.3 & 2.8 \\
Leu & 3.1 & 2.8 & 3.1 & 3.5 & 3.2 & 3.0 & 2.8 \\
Tyr & 1.8 & 3.2 & 1.0 & 1.5 & 0.0 & 0.0 & 2.1 \\
Phe & 1.6 & 3.1 & 1.8 & 1.6 & 1.9 & 1.8 & 2.2 \\
$\gamma \mathrm{ABA}$ & 0.5 & 0.0 & 0.0 & 0.5 & 0.0 & 0.0 & 0.0 \\
Orn & 11.7 & 9.1 & 9.7 & 12.4 & 10.3 & 11.7 & 15.8 \\
Lys & 3.6 & 1.9 & 2.6 & 2.9 & 4.0 & 3.1 & 3.9 \\
His & 4.5 & 2.6 & 2.9 & 4.0 & 3.2 & 3.7 & 3.9 \\
Arg & 0.0 & 1.0 & 0.3 & 1.4 & 0.6 & 3.6 & 0.0 \\
Total AA & 8.34 & 1.6 & 11.37 & 12.88 & 15.02 & 2.6 & 5.21 \\
( $\mu$ mol/L) & & & & & & & \\
Depth & 2.9 & 62.8 & 6.9 & 107.0 & 144.9 & 154.4 & 201.0 \\
(mbsf) & & & & & & & \\
DOC & 4.2 & 3.1 & 7.9 & 15.4 & 73.8 & 33.6 & n.d. \\
(mg/L) & & & & & & & \\
AA/DOC & 9.56 & 2.64 & 2.39 & 4.45 & 1.01 & 0.38 & n.d. \\
\hline Individual amino acids are given & & & & & & & \\
\end{tabular}

Individual amino acids are given in $\mathrm{mol} \%$

Table 7. Amino acids (AA) and amino sugars (AS) in sediment, Hole 651A.

\begin{tabular}{|c|c|c|c|c|c|c|c|c|c|c|c|c|c|c|}
\hline Hole & $653 \mathrm{~A}$ & $653 \mathrm{~B}$ & $653 \mathrm{~B}$ & $653 \mathrm{~B}$ & $653 \mathrm{~B}$ & $653 \mathrm{~B}$ & $653 \mathrm{~B}$ & $653 \mathrm{~A}$ & $653 \mathrm{~B}$ & $653 \mathrm{~B}$ & $653 \mathrm{~B}$ & $653 \mathrm{~B}$ & $653 \mathrm{~B}$ & $653 \mathrm{~B}$ \\
\hline Sample & $3 R-1$ & $4 R-3$ & $12 \mathrm{R}-1$ & $17 \mathrm{R}-3$ & $18 \mathrm{R}-5$ & $23 \mathrm{R}-1$ & $37 \mathrm{R}-2$ & $37 R-4$ & $39 \mathrm{R}-2$ & $39 \mathrm{R}-3$ & $40 \mathrm{R}-2$ & $40 \mathrm{R}-4$ & $41 R-2$ & $41 R-4$ \\
\hline Asp & 12.1 & 13.0 & 8.0 & 7.7 & 7.2 & 8.1 & 6.6 & 5.8 & 8.5 & 9.4 & 2.0 & 8.6 & 4.5 & 5.1 \\
\hline Thr & 2.9 & 3.6 & 1.2 & 2.0 & 1.2 & 4.3 & 3.3 & 1.0 & 5.4 & 8.3 & 0.0 & 2.8 & 0.7 & 0.4 \\
\hline Ser & 2.0 & 2.5 & 1.6 & 1.0 & 1.5 & 4.5 & 6.9 & 2.2 & 15.8 & 10.7 & 12.2 & 7.0 & 1.4 & 15.6 \\
\hline Glu & 3.7 & 8.1 & 5.2 & 4.7 & 4.5 & 12.5 & 14.9 & 13.8 & 3.7 & 6.9 & 4.0 & 11.5 & 4.3 & 6.6 \\
\hline Gly & 8.7 & 11.2 & 9.5 & 1.5 & 10.3 & 13.6 & 16.9 & 24.4 & 16.5 & 19.1 & $58.9^{*}$ & 17.4 & $60.8^{*}$ & $39.8^{*}$ \\
\hline Ala & 6.1 & 8.0 & 5.9 & 15.0 & 6.6 & 9.3 & 15.1 & 7.4 & 6.9 & 6.1 & & 7.0 & & \\
\hline Val & 4.4 & 6.2 & 3.2 & 5.7 & 4.7 & 4.4 & 6.2 & 5.8 & 3.6 & 5.6 & 2.1 & 3.5 & 2.7 & 5.2 \\
\hline Met & 0.2 & 0.4 & 1.6 & 0.0 & 0.7 & 0.0 & 0.0 & 13.3 & 0.0 & 0.0 & 0.0 & 0.0 & 0.0 & 0.0 \\
\hline Ile & 1.4 & 3.2 & 1.3 & 3.4 & 1.7 & 2.7 & 2.1 & 2.1 & 3.0 & 2.6 & 2.7 & 0.9 & 2.9 & 2.6 \\
\hline Leu & 4.1 & 4.9 & 2.1 & 6.7 & 4.0 & 5.0 & 3.8 & 3.3 & 3.8 & 4.2 & 5.9 & 3.4 & 5.3 & 4.1 \\
\hline Tyr & 0.2 & 1.0 & 0.4 & 0.6 & 0.3 & 0.0 & 1.0 & 0.6 & 1.3 & 3.8 & 0.0 & 0.0 & 0.0 & 0.0 \\
\hline Phe & 1.6 & 2.3 & 1.0 & 2.1 & 1.2 & 1.6 & 2.0 & 1.4 & 1.1 & 3.3 & 0.0 & 0.0 & 0.0 & 0.0 \\
\hline$\beta$ Ala & 17.8 & 9.7 & 14.2 & 12.8 & 16.2 & 7.8 & 2.3 & 5.8 & 3.0 & 0.0 & 0.0 & 4.0 & 0.0 & 0.0 \\
\hline$\gamma \mathrm{ABA}$ & 17.6 & 11.7 & 27.3 & 22.8 & 28.0 & 13.4 & 2.4 & 6.0 & 2.0 & 3.1 & 6.8 & 5.3 & 1.4 & 1.5 \\
\hline Orn & 1.9 & 1.8 & 2.8 & 2.2 & 2.7 & 5.0 & 3.1 & 2.1 & 12.4 & 5.3 & 0.8 & 18.8 & 10.6 & 13.8 \\
\hline Lys & 6.5 & 5.9 & 5.1 & 7.3 & 5.3 & 4.8 & 2.7 & 2.6 & 4.7 & 5.3 & 0.0 & 6.0 & 0.0 & 0.0 \\
\hline His & 2.7 & 1.7 & 5.2 & 2.5 & 2.9 & 0.0 & 9.2 & 2.5 & 5.0 & 0.0 & 0.0 & 0.0 & 2.4 & 2.9 \\
\hline Arg & 6.1 & 4.9 & 3.2 & 2.1 & 0.7 & 2.8 & 1.4 & 0.0 & 3.8 & 6.4 & 4.7 & 3.9 & 3.0 & 2.4 \\
\hline $\begin{array}{l}\text { Total AA } \\
(\mu \mathrm{mol} / \mathrm{g})\end{array}$ & 0.58 & 0.97 & 0.46 & 0.21 & 0.41 & 0.09 & 0.16 & 0.08 & 0.09 & 0.07 & 0.02 & 0.04 & 0.04 & 0.04 \\
\hline $\mathrm{GlcNH}_{2}$ & 48.2 & 92.8 & 47.0 & 33.1 & 47.9 & 4.7 & 24.2 & 3.1 & 10.2 & 0.0 & 0.0 & 0.0 & 0.0 & 0.0 \\
\hline GalNH $_{2}$ & 29.8 & 52.1 & 17.5 & 11.1 & 9.4 & 0.0 & 0.0 & 0.0 & 0.0 & 0.0 & 0.0 & 0.0 & 0.0 & 0.0 \\
\hline $\begin{array}{l}\text { Total AS } \\
(\mathrm{nmol} / \mathrm{g})\end{array}$ & 78.0 & 144.9 & 64.5 & 44.2 & 57.3 & 4.7 & 24.2 & 3.1 & 10.2 & 0.0 & 0.0 & 0.0 & 0.0 & 0.0 \\
\hline $\begin{array}{l}\text { Depth } \\
\text { (mbsf) }\end{array}$ & 10.8 & 23.3 & 97.9 & 149.0 & 162.2 & 204.6 & 339.9 & 342.9 & 359.7 & 361.8 & 369.3 & 372.3 & 378.4 & 381.4 \\
\hline $\begin{array}{l}\mathrm{AA} / \mathrm{C}_{\mathrm{org}} \\
(\%)\end{array}$ & n.d. & n.d. & n.d. & n.d. & 0.15 & n.d. & n.d. & n.d. & n.d. & n.d. & n.d. & n.d. & n.d. & n.d. \\
\hline $\begin{array}{l}\text { AS/C } C_{\text {org }} \\
(\%)\end{array}$ & n.d. & n.d. & n.d. & n.d. & 0.03 & n.d. & n.d. & n.d. & n.d. & n.d. & n.d. & n.d. & n.d. & n.d. \\
\hline
\end{tabular}

* Gly + Ala 
Table 8. Amino acids (AA) and amino sugars (AS) in sediment, Site 653.

\begin{tabular}{|c|c|c|c|c|c|c|c|c|c|c|c|c|c|c|c|c|c|c|c|c|c|}
\hline Sample & $1 \mathrm{H}-4$ & $2 \mathrm{H}-5$ & $3 \mathrm{H}-4$ & $4 \mathrm{H}-3$ & $5 \mathrm{H}-5$ & $6 \mathrm{H}-4$ & $6 \mathrm{H}-5$ & $7 \mathrm{H}-3$ & $8 \mathrm{H}-2$ & $9 X-2$ & $12 \mathrm{X}-3$ & $13 \mathrm{X}-4$ & $14 X-2$ & $16 \mathrm{X}-3$ & $17 X-3$ & $18 X-4$ & $19 \mathrm{X}-4$ & $20 \mathrm{X}-3$ & $21 X-3$ & $22 \mathrm{X}-3$ & $23 \mathrm{X}-3$ \\
\hline Asp & 8.3 & 7.3 & 11.1 & 5.1 & 14.4 & 11.9 & 12.4 & 11.0 & 6.0 & 17.4 & 13.6 & 11.1 & 14.8 & 12.1 & 13.6 & 11.0 & 10.5 & 10.3 & 11.8 & 9.4 & 8.7 \\
\hline Thr & 2.5 & 1.1 & 1.7 & 1.9 & 2.5 & 1.3 & 1.6 & 1.1 & 1.1 & 1.9 & 2.1 & 1.1 & 0.6 & 0.0 & 3.0 & 2.7 & 0.6 & 0.8 & 3.9 & 4.0 & 2.7 \\
\hline Ser & 3.1 & 2.7 & 2.0 & 5.1 & 4.5 & 4.9 & 4.5 & 1.5 & 4.7 & 1.2 & 6.4 & 5.0 & 0.4 & 2.2 & 5.9 & 6.7 & 3.9 & 8.3 & 4.6 & 12.5 & 8.7 \\
\hline Glu & 5.3 & 4.0 & 4.0 & 4.8 & 9.4 & 8.8 & 8.9 & 9.2 & 4.3 & 13.6 & 11.8 & 9.4 & 9.0 & 15.9 & 15.4 & 14.5 & 13.9 & 13.6 & 18.3 & 11.1 & 9.5 \\
\hline Gly & 5.0 & 6.0 & 2.6 & 5.3 & 12.0 & 9.4 & 10.0 & 5.4 & 7.1 & 12.4 & 11.7 & 9.4 & 8.6 & 13.9 & 10.3 & 12.3 & 10.8 & 15.9 & 11.4 & 13.3 & 8.0 \\
\hline Ala & 7.8 & 4.4 & 6.2 & 7.3 & 9.3 & 7.8 & 9.1 & 8.3 & 4.6 & 7.5 & 9.9 & 5.9 & 11.7 & 10.1 & 11.0 & 12.8 & 11.9 & 11.4 & 13.3 & 11.4 & 20.2 \\
\hline Val & 3.5 & 2.8 & 3.4 & 3.0 & 5.3 & 4.7 & 5.2 & 4.2 & 3.6 & 7.4 & 5.8 & 6.8 & 7.0 & 8.3 & 5.0 & 7.4 & 6.2 & 8.6 & 8.1 & 6.3 & 6.0 \\
\hline Met & 1.0 & 0.0 & 0.0 & 0.0 & 2.5 & 0.0 & 0.0 & 0.0 & 0.7 & 0.0 & 0.0 & 1.9 & 0.0 & 0.0 & 0.0 & 0.0 & 0.0 & 0.0 & 0.0 & 0.7 & 0.0 \\
\hline Ile & 1.6 & 1.1 & 1.1 & 1.3 & 2.3 & 2.3 & 2.2 & 1.2 & 2.1 & 2.2 & 1.7 & 3.4 & 2.3 & 1.8 & 2.0 & 2.1 & 1.9 & 2.5 & 2.7 & 2.6 & 1.7 \\
\hline Leu & 2.4 & 2.4 & 3.2 & 2.8 & 3.4 & 3.2 & 3.5 & 2.3 & 3.1 & 4.6 & 3.5 & 3.5 & 4.8 & 4.2 & 4.5 & 3.9 & 4.3 & 3.6 & 5.2 & 4.8 & 4.1 \\
\hline Tyr & 0.5 & 0.3 & 0.2 & 0.7 & 0.6 & 1.9 & 0.9 & 1.1 & 0.0 & 0.8 & 3.4 & 1.1 & 0.0 & 0.0 & 1.5 & 0.9 & 0.7 & 0.6 & 0.9 & 2.3 & 0.4 \\
\hline Phe & 0.9 & 0.7 & 1.3 & 1.5 & 1.9 & 1.6 & 1.5 & 1.8 & 1.5 & 1.5 & 2.1 & 1.6 & 0.8 & 0.0 & 2.3 & 1.4 & 1.4 & 1.1 & 1.0 & 2.7 & 2.5 \\
\hline$\beta$ Ala & 17.9 & 16.5 & 17.0 & 15.4 & 8.1 & 9.4 & 9.9 & 13.8 & 13.8 & 8.5 & 5.1 & 8.6 & 12.2 & 8.3 & 7.7 & 5.4 & 7.3 & 3.6 & 5.4 & 3.0 & 7.8 \\
\hline$\gamma \mathrm{ABA}$ & 26.5 & 33.2 & 35.8 & 33.2 & 13.8 & 16.2 & 17.4 & 30.5 & 29.6 & 13.4 & 8.7 & 15.3 & 18.9 & 7.4 & 7.9 & 5.5 & 10.8 & 4.7 & 5.1 & 2.9 & 5.7 \\
\hline Orn & 2.6 & 4.5 & 0.9 & 4.3 & 3.8 & 5.2 & 4.5 & 2.1 & 11.2 & 2.2 & 5.2 & 6.8 & 1.6 & 3.1 & 2.6 & 4.8 & 4.2 & 7.5 & 2.1 & 7.3 & 6.7 \\
\hline Lys & 4.0 & 0.8 & 4.5 & 4.2 & 3.5 & 3.8 & 4.5 & 4.5 & 4.2 & 4.2 & 5.9 & 3.5 & 4.5 & 6.0 & 4.5 & 4.7 & 6.2 & 4.5 & 3.5 & 3.9 & 4.7 \\
\hline $\mathrm{His}$ & 2.2 & 3.0 & 1.9 & 1.6 & 2.8 & 6.1 & 1.6 & 0.9 & 1.1 & 1.3 & 3.0 & 1.1 & 1.0 & 6.6 & 2.1 & 3.8 & 4.6 & 3.0 & 0.8 & 1.8 & 2.5 \\
\hline Arg & 5.0 & 9.1 & 2.9 & 2.3 & 0.0 & 1.4 & 2.1 & 1.0 & 1.5 & 0.0 & 0.0 & 4.6 & 1.4 & 0.0 & 0.8 & 0.0 & 0.9 & 0.0 & 2.1 & 0.1 & 0.0 \\
\hline $\begin{array}{l}\text { Total AA } \\
(\mu \mathrm{mol} / \mathrm{g})\end{array}$ & 1.06 & 0.65 & 0.45 & 0.7 & 0.46 & 0.3 & 0.27 & 0.33 & 0.24 & 0.31 & 0.17 & 0.12 & 0.19 & 0.2 & 0.21 & 0.17 & 0.16 & 0.1 & 0.12 & 0.23 & 0.09 \\
\hline $\mathrm{GlcNH}_{2}$ & 170.0 & 74.1 & 16.1 & 23.0 & 45.2 & 35.1 & 37.3 & 25.6 & 177.4 & 25.3 & 25.3 & 124.9 & 26.3 & 30.7 & 17.1 & 4.2 & 13.9 & 21.7 & 9.0 & 28.1 & 21.6 \\
\hline $\mathrm{GalNH}_{2}$ & 85.7 & 32.9 & 4.3 & 5.4 & 11.8 & 6.8 & 10.1 & 6.3 & 10.9 & 0.0 & 1.0 & 5.2 & 3.2 & 0.0 & 0.0 & 0.0 & 0.0 & 0.0 & 0.0 & 0.0 & 0.0 \\
\hline $\begin{array}{l}\text { Total AS } \\
(\mathrm{nmol} / \mathrm{g})\end{array}$ & 255.7 & 107.0 & 20.4 & 28.4 & 57.0 & 41.9 & 47.4 & 31.9 & 188.3 & 25.3 & 26.3 & 130.1 & 29.5 & 30.7 & 17.1 & 4.2 & 13.9 & 21.7 & 9.0 & 28.1 & 21.6 \\
\hline $\begin{array}{l}\text { Depth } \\
\text { (mbsf) }\end{array}$ & 4.9 & 13.4 & 22.0 & 31.5 & 44.0 & 51.9 & 52.6 & 59.4 & 66.9 & 77.1 & 107.0 & 118.0 & 124.1 & 144.9 & 154.4 & 165.5 & 174.2 & 182.4 & 191.4 & 201.0 & 210.5 \\
\hline $\begin{array}{l}\mathrm{AA} / \mathrm{C}_{\text {org }} \\
(\%)\end{array}$ & 0.55 & 2.47 & 1.3 & 1.35 & 0.38 & n.d. & 0.25 & 0.29 & 0.19 & 0.22 & n.d. & n.d. & n.d. & n.d. & 0.72 & 0.3 & n.d. & n.d. & n.d. & n.d. & n.d. \\
\hline $\begin{array}{l}\mathrm{AS} / \mathrm{C}_{\text {org }} \\
(\%)\end{array}$ & 0.14 & 0.49 & 0.08 & 0.07 & 0.06 & n.d. & 0.05 & 0.03 & 0.18 & 0.02 & n.d. & n.d. & n.d. & n.d. & 0.07 & 0.01 & n.d. & n.d. & n.d. & n.d. & n.d. \\
\hline
\end{tabular}

Individual amino acids are given in mol\%; $\mathrm{GlcNH}_{2}=$ glucoseamine, $\mathrm{GalNH}_{2}=$ galactoseamine, $\mathrm{AA} / \mathrm{C}_{\mathrm{org}}=$ percentage of amino acids of organic carbon, $\mathrm{AS} / \mathrm{C}_{\mathrm{org}}=$ percentage of amino sugars of organic carbon 

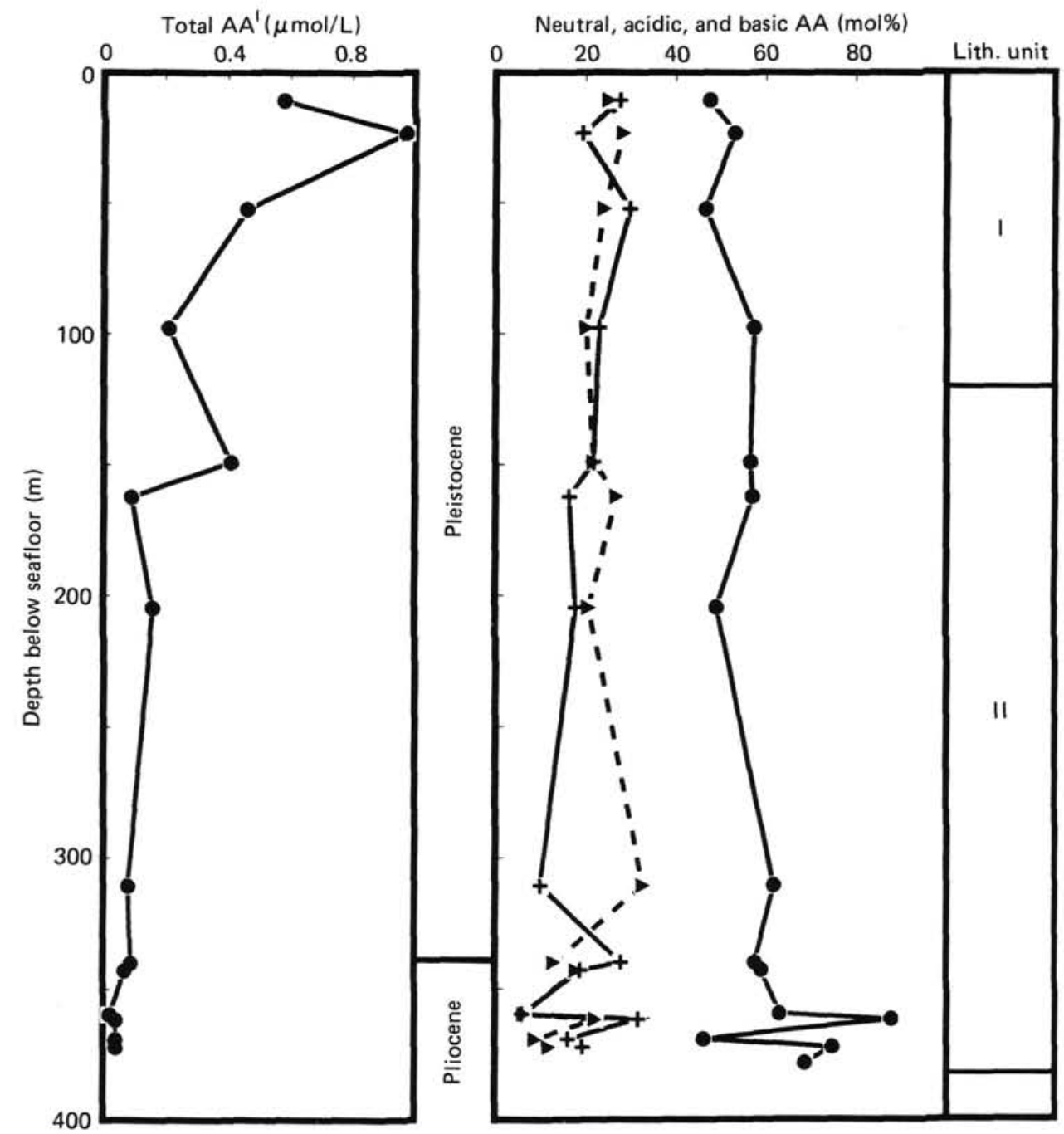

Figure 3. Downhole variability in the total amount of amino acids (AA) in sediments of Hole 651A (left). The contributions of neutral (dots, solid line), acidic (triangles, broken line), and basic amino acids (pluses, solid line) are depicted to the right. Neutral amino acids are threonine, serine, glycine, alanine, valine, isoleucine, and leucine. Acidic amino acids are aspartic and glutamic acid, and basic amino acids are arginine, histidine, lysine, and ornithine.

$\gamma$-ABA can also result from chemical diagenesis of glutamic acid (Glu) (Itihara, 1973; Schroeder, 1975).

The Glu/ $\beta$-ABA and Asp/ $\beta$-Ala ratios for sediment samples from Sites 651 and 653 are shown in Figures 5 and 6, respectively. $\beta$-Ala could only be detected in one sample below 360 mbsf at Site 651A. Quaternary samples display similar curves for these ratios at both sites, and the Glu/ $\gamma$-ABA and Asp/ $\beta$ Ala ratios are low. We believe that these results may be an indication of high microbial activity in the young sediments, because degradation of primary amino compounds increases the abundance of these degradation products. Near the Pliocene/ Pleistocene boundary (Samples 653B-9X-2 and 651A-37R-2) both ratios increase; this increase may indicate that amino acids bound to the organic matrix of biogenic calcite comprise a major partion of the total amino acid fraction, which is only 0.31 and $0.16 \mu \mathrm{mol} / \mathrm{g}$ of sediment (less than 0.02 of organic carbon; Table 8). Amino acid spectra of foraminifers are found to be dominated by aspartic acid, glycine, and glutamic acid (King and Hare, 1972), and no $\gamma$-ABA and $\beta$-Ala was detected in cleaned shell material from deep-sea sediments as old as $40.000 \mathrm{yr}$ (Schroeder, 1975). According to these observations, the acidic amino acids embedded in the protein matrix of calcitic shell ma- terial are not degraded to $\gamma$-Ala or $\beta$-ABA during early diagenesis, but instead are protected from bacterial or thermal degradation, until recrystallization of the carbonate tests sets them free.

Ornithine also represents another nonprotein amino acid, which may originate from either abiotic and microbial degradation of arginine (Degens, 1970) or from constituents of bacterial cell walls (Goossens et al., 1986; Kandler, 1979, 1981). In contrast to the results from Site 653, high percentages of ornithine are found in the dolomitic Pliocene strata of Hole 651A. This finding may be ascribed to higher thermal stability of ornithine compared to other amino acids (Khan and Sowden, 1971). Low concentrations of $\gamma$-Aba and $\beta$-Ala were found in the dissolved amino acids of interstitial waters, whereas ornithine was abundant in all analyzed samples (Table 3 ).

\section{Carbohydrates}

Concentrations of carbohydrates in sediment are given in Tables 9 and 10 for samples from Site 651 and Site 653, and sugar values for pore waters are summarized in Table 11. Total amounts of sugars in sediments range from 0.21 to $1.82 \mu \mathrm{mol} / \mathrm{g}$, and in pore water from 5.01 to $78.42 \mu \mathrm{mol} / \mathrm{L}$. 


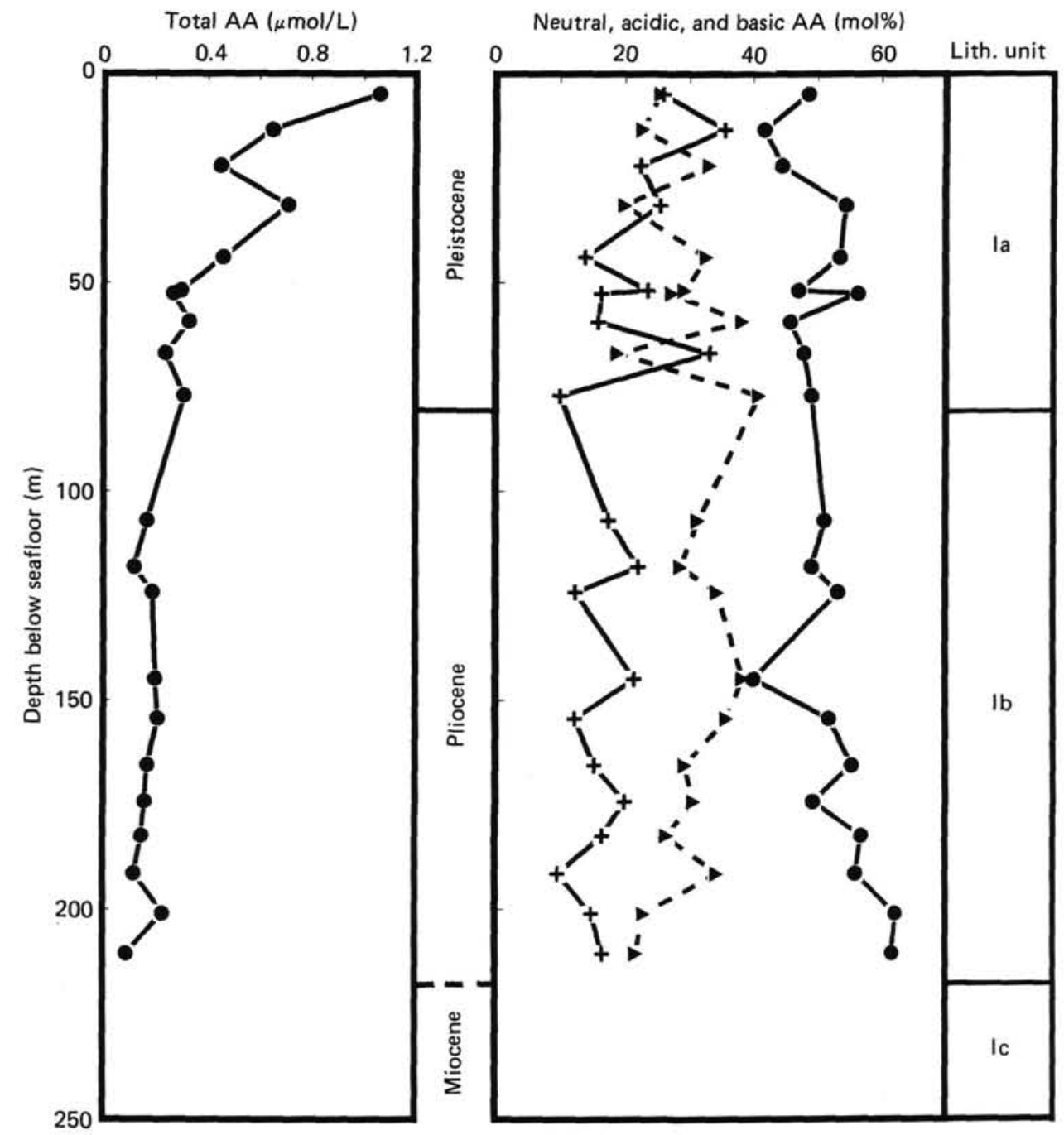

Figure 4. Downhole variability in the total amount of amino acids (AA) in sediments of Site 653 (left). The contributions of neutral (dots, solid line), acidic (triangles, broken line), and basic amino acids (pluses, solid line) are depicted to the right. Neutral amino acids are threonine, serine, glycine, alanine, valine, isoleucine, and leucine. Acidic amino acids are aspartic and glutamic acid, and basic amino acids are arginine, histidine, lysine, and ornithine.

The downhole distribution pattern of total sugars in sediment samples from Site 653 (Fig. 7) resembles that of total amino acids. Sample 653B-20X-3 (182.4 mbsf) is an exception because sugar contents are higher than in other Pliocene samples. Figure 7 also gives the variations in the galactose/fructose ratio. Galactose, together with mannose, rhamnose and xylose, is abundant in fresh plankton (Cowie and Hedges, 1984; Hecky et al., 1973; Degens and Mopper, 1975) and is, with mannose and glucose, the main sugar in bacterial cell walls (Kandler and König, 1978). Galactose is also a component of the heteropolysaccharides of land plants. Fructose has been found to be a major constituent in the sugar fraction of old sediments (Michaelis et al., 1986; Mopper et al., 1978; Emeis et al., 1987). Analyses of recent sediments from a variety of environments revealed low fructose contents, however, and these studies suggest high stability of fructose relative to other carbohydrates (Mopper, 1977; Bartsch, 1987). A preferential association of fructose with polyvalent metals, particularly with transition metals, has been described by Charley et al. (1963) and Barker et al. (1974). Degens and Mopper (1976) proposed this association as a stabilizing mechanism that preserves both metals and fructose. An enrichment of this sugar was also found in the humic matter of river water by Seifert and lttekkot (1985), who believe that the incorporation of fructose in macromolecules and chelates protects this sugar against early diagenetic transformation in the geological environment. A feasible source of fructose is the abiotic epimerization of glucose in weakly alkaline solutions (Mopper et al., 1980). Laboratory experiments in seawater at a pH of 8.1 showed that this epimerization is significant especially at temperatures exceeding early diagenetic levels.

The galactose/fructose ratio can thus be used as an indicator of the relative freshness of the organic matter. Sediments that contain only weakly degraded organic material will have high galactose/fructose ratios, while those containing organic material exposed to biotic or thermal degradation processes for longer periods of time have low ratios. Furthermore, the galactose/fructose ratio should decrease with time as a function of differential degradation and as an effect of higher stability of fructose as compared to galactose and other carbohydrate monomers.

The galactose/fructose ratios for samples from Core $651 \mathrm{~A}$ are listed in Table 9. The ratio decreases with depth, and a minimum in Sample 18R-5 coincides with a low carbonate value. As shown in Figure 7, the curve of the galactose/fructose ratios for 


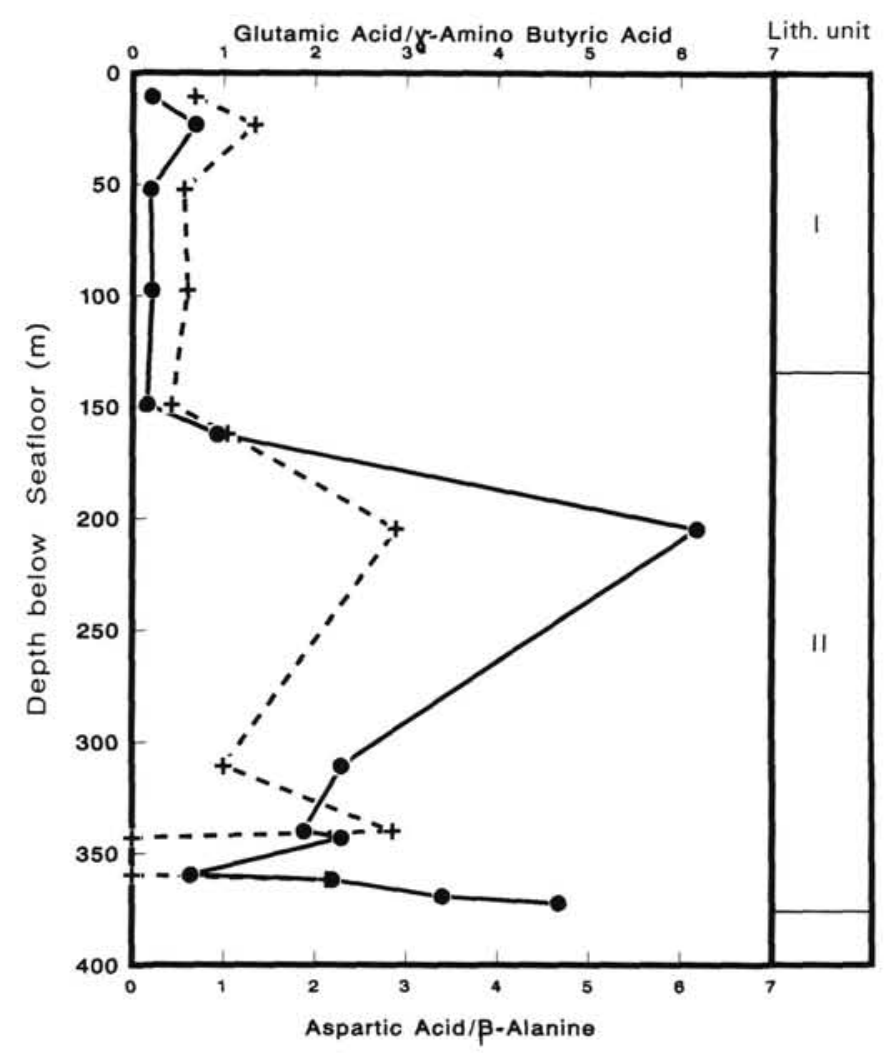

Figure 5. Depth plots of the ratios of glutamic acid to $\gamma$-amino butyric acid (dots, solid line) and aspartic acid to $\beta$-alanine (pluses, broken line), which are two indicators for microbial degradation in sediments of Hole 651A. Low values show advanced degradation, while high values may denote organic matter of terrestrial origin. See text for explanation.

Hole 653B is in good agreement with the distribution of total carbohydrates: samples high in organic carbon have higher ratios, because they contain more labile galactose when compared to samples low in organic carbon. Exceptions are the Samples $653 \mathrm{~B}-4 \mathrm{H}-3$ and $-8 \mathrm{H}-2$, which are characterized by a low carbonate content. In these samples the organic material may derive from terrigenous sources and may have been exposed to intensive decomposition prior to deposition. The differences among the other samples are presumably caused by factors such as variations in the quantity and quality of primary production and by different sedimentation rates, which affect the duration and degree of bacterial decomposition.

An increase in the dominance of fructose and glucose with depth in the carbohydrates of interstitial waters is observed at both sites. Previous studies of carbohydrates in interstitial waters from DSDP Leg 93 revealed similar trends (Emeis et al., 1987). Glucose and fructose are found to be the major sugars in subsoil waters (Spitzy, 1982) and in the free dissolved fraction of seawater (Mopper et al., 1980; Ittekkot et al., 1981). These observations underscore their relative stability and comparatively long residence time in both particulate and dissolved form. The fructose concentrations in the dissolved carbohydrate fraction are higher in Hole 651A than in Hole 653B. Whereas the fructose maximum in Sample 653B-6H-4 may be correlative with a high value for dissolved iron (Table 4), we are not able to explain the observed trends in dissolved carbohydrates with the available data. It appears that metal stabilization does not play any significant role in preserving dissolved sugars, as will be briefly discussed below.

Analyses of selected minor and trace constituents in the interstitial waters of Sites 651 and 653 are listed in Tables 3 and 4 .

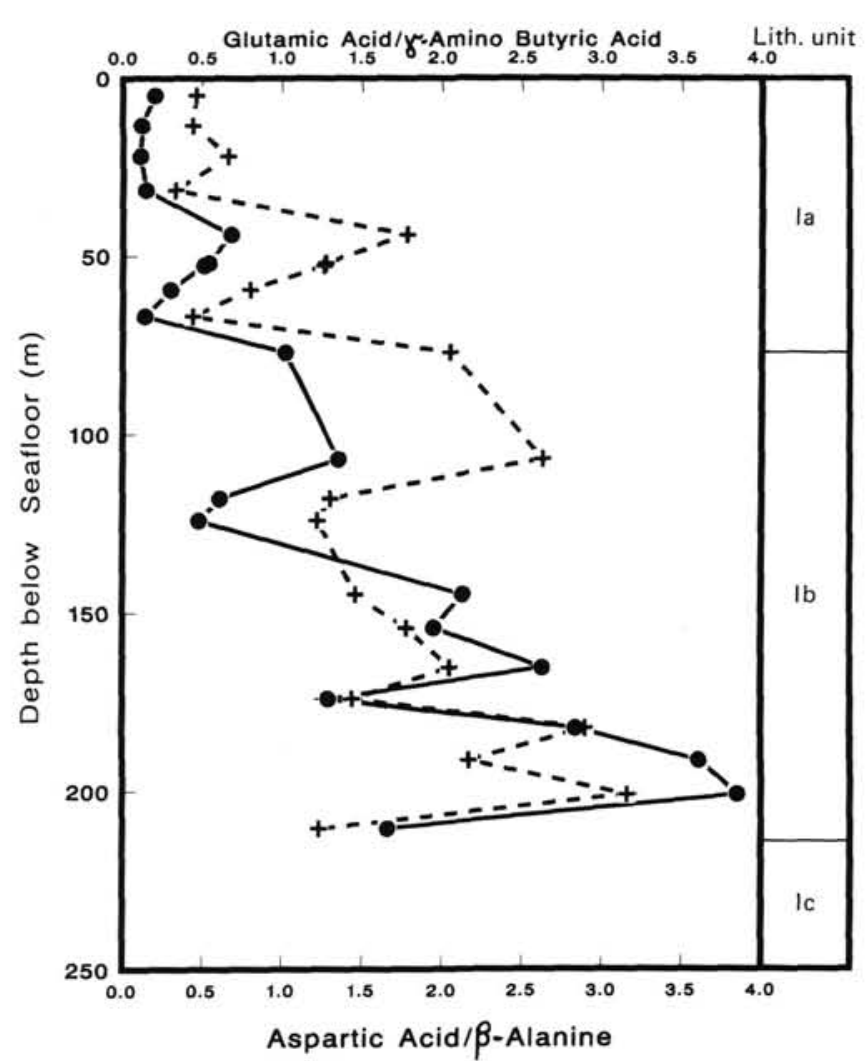

Figure 6. Depth plots of the ratios of glutamic acid to $\gamma$-amino butyric acid (dots, solid line) and aspartic acid to $\beta$-alanine (pluses, broken line), which are two indicators for microbial degradation in sediments of Site 653 . Low values show advanced degradation, while high values may denote organic matter of terrestrial origin. See text for explanation.

Table 9. Carbohydrates in sediment, Hole 651A.

\begin{tabular}{lrrrrr}
\hline Sample & $3 R-1$ & $12 R-1$ & $17 R-3$ & $18 R-5$ & $40 R-2$ \\
\hline Rha & 5.9 & 3.9 & 5.5 & 3.2 & 0.0 \\
Man & 11.3 & 5.5 & 6.6 & 1.5 & 4.1 \\
Fru & 4.6 & 19.4 & 20.9 & 50.2 & 31.5 \\
Ara & 20.5 & 11.4 & 13.0 & 2.9 & 1.3 \\
Fuc & 10.1 & 5.5 & 5.6 & 1.8 & 1.0 \\
Gal & 17.7 & 13.1 & 11.8 & 0.7 & 5.0 \\
Xyl & 11.5 & 6.8 & 7.6 & 1.0 & 0.5 \\
Glc & 18.5 & 34.5 & 29.1 & 38.8 & 56.6 \\
Total & 0.98 & 0.56 & 0.91 & 1.42 & 0.23 \\
( $\mu$ mol/g) & & & & & \\
Depth & 10.8 & 97.9 & 149.0 & 162.2 & 369.3 \\
(mbsf) & & & & & \\
Gal/Fru & 3.88 & 0.68 & 0.56 & 0.01 & 0.16 \\
\hline
\end{tabular}

Individual sugars are given in $\mathrm{mol} \%$.

We had hoped to find indications of unusual trace metal abundances, in particular of manganese and iron. We expected to see effects of halmyrolysis of volcanic ash and of alteration of the relatively young basalts at Site 651 , and we had expected to verify correlations of dissolved metals with chelating agents in DOC. Even though the abundances vary widely for both elements, we failed to recognize any meaningful trend in the data. This result may be attributable to some extent to inadequacies in sample preparation (squeezing at laboratory temperatures and under oxygen on less than clean benches) and to the effects of complexing agents (such as DOC and certain carbohydrates) on 
Table 10. Carbohydrates in sediment, Site 653.

\begin{tabular}{|c|c|c|c|c|c|c|c|c|c|c|c|c|c|}
\hline Sample & $1 \mathrm{H}-4$ & $2 \mathrm{H}-5$ & $3 \mathrm{H}-4$ & $4 \mathrm{H}-3$ & $5 \mathrm{H}-5$ & $6 \mathrm{H}-4$ & $6 \mathrm{H}-5$ & $8 \mathrm{H}-2$ & $9 X-2$ & $16 \mathrm{X}-3$ & $18 X-4$ & $20 \mathrm{X}-3$ & $23 X-3$ \\
\hline Rha & 7.3 & 3.0 & 5.7 & 3.1 & 4.2 & 4.4 & 4.9 & 3.9 & 4.5 & 0.0 & 1.3 & 9.8 & 11.9 \\
\hline Rib & 7.9 & 0.0 & 3.0 & 0.0 & 0.0 & 0.0 & 0.0 & 0.0 & 0.0 & 0.0 & 0.0 & 0.0 & 0.0 \\
\hline Man & 9.0 & 10.4 & 15.1 & 10.5 & 18.1 & 13.7 & 22.7 & 15.0 & 20.4 & 7.2 & 4.6 & 15.3 & 4.7 \\
\hline Fru & 5.6 & 6.5 & 7.2 & 22.6 & 3.1 & 17.4 & 3.7 & 10.2 & 3.8 & 23.3 & 18.0 & 4.4 & 14.6 \\
\hline Ara & 13.2 & 14.9 & 15.2 & 17.8 & 16.6 & 1.4 & 12.7 & 17.4 & 14.0 & 11.9 & 9.3 & 6.4 & 14.1 \\
\hline Fuc & 8.8 & 5.0 & 7.2 & 11.6 & 12.8 & 10.1 & 12.3 & 11.9 & 13.5 & 17.9 & 12.6 & 10.6 & 8.1 \\
\hline Gal & 18.2 & 16.5 & 14.6 & 10.6 & 18.4 & 11.1 & 14.1 & 11.7 & 11.9 & 12.0 & 5.9 & 18.2 & 1.3 \\
\hline Xyl & 9.7 & 10.6 & 11.1 & 5.6 & 11.4 & 9.3 & 11.9 & 9.2 & 9.1 & 4.1 & 4.6 & 9.4 & 1.0 \\
\hline Glc & 20.4 & 33.1 & 20.9 & 18.3 & 15.5 & 32.6 & 17.7 & 20.7 & 22.9 & 23.6 & 43.8 & 25.9 & 44.3 \\
\hline $\begin{array}{l}\text { Total } \\
(\mu \mathrm{mol} / \mathrm{g})\end{array}$ & 1.82 & 1.5 & 0.53 & 1.44 & 0.56 & 0.3 & 0.69 & 0.52 & 0.65 & 0.32 & 0.44 & 1.24 & 0.21 \\
\hline $\begin{array}{l}\text { Depth } \\
\text { (mbsf) }\end{array}$ & 4.9 & 13.4 & 22.0 & 31.5 & 44.0 & 51.9 & 52.6 & 66.9 & 77.1 & 144.9 & 165.5 & 182.4 & 210.5 \\
\hline $\begin{array}{l}\mathrm{CHO} / \mathrm{C}_{\text {org }} \\
(\%)\end{array}$ & 1.1 & 8.0 & 2.1 & 4.4 & 0.6 & n.d. & 0.8 & 0.6 & 0.7 & n.d. & 1.1 & n.d. & n.d. \\
\hline
\end{tabular}

Individual sugars are given in $\mathrm{mol} \%$

Table 11. Carbohydrates in interstitial water, Sites 651 and 653.

\begin{tabular}{lrrrrrrr}
\hline \multicolumn{1}{c}{ Hole } & $653 \mathrm{~B}$ & $653 \mathrm{~B}$ & $653 \mathrm{~B}$ & $653 \mathrm{~B}$ & $651 \mathrm{~A}$ & $651 \mathrm{~A}$ & $651 \mathrm{~A}$ \\
Sample & \multicolumn{1}{c}{$3 \mathrm{H}-4$} & $5 \mathrm{H}-5$ & $8 \mathrm{H}-2$ & $13 \mathrm{X}-4$ & $3 \mathrm{R}-1$ & $7 \mathrm{R}-2$ & $18 \mathrm{R}-5$ \\
\hline Rha & 9.4 & 5.1 & 7.6 & 2.4 & 0.0 & 0.0 & 0.0 \\
Man & 14.8 & 11.0 & 12.7 & 1.9 & 3.1 & 0.7 & 0.5 \\
Fru & 2.0 & 9.2 & 17.2 & 13.1 & 39.9 & 47.2 & 52.8 \\
Ara & 7.1 & 5.7 & 13.9 & 0.0 & 2.3 & 3.7 & 0.3 \\
Fuc & 7.1 & 6.8 & 7.2 & 0.0 & 7.2 & 3.4 & 2.3 \\
Gal & 13.7 & 11.2 & 4.9 & 7.9 & 5.9 & 4.6 & 0.8 \\
Xyl & 6.0 & 3.9 & 10.2 & 1.5 & 2.5 & 0.8 & 0.6 \\
Glc & 40.0 & 47.2 & 26.4 & 73.2 & 39.1 & 39.6 & 42.7 \\
Total & 5.01 & 7.76 & 17.08 & 7.5 & 24.8 & 25.38 & 78.42 \\
( $\mu$ mol/L) & & & & & & & \\
Depth & 22.0 & 44.0 & 66.9 & 118.0 & 10.8 & 52.4 & 162.2 \\
(mbsf) & & & & & & & \\
\hline
\end{tabular}

Individual sugars are given in $\mathrm{mol} \%$

metal solubility. These organic substances, as was pointed out above, are liberated during various stages of sediment and organic matter diagenesis, and are pronounced near organic-rich horizons. The available data preclude speculation on the mechanisms of organic matter/metal interactions, however, and we are not at all certain whether the high metal concentrations in some samples are related to dissolved organic matter.

\section{CONCLUSIONS}

Downhole distribution of labile organic components in sediments (amino acids and sugars, and carbohydrates) seems to be affected by differences in source materials and their resistance to microbial degradation, by sedimentation rate, and by thermal maturity, rather than by organic matter abundances in the sediment. Comparison of distributions of these compound classes from Sites 651 and 653, where sedimentation rates differ by a factor of two, and which have different thermal histories, shows that increased heat flow at Site 651 resulted in preferential depletion of amino sugars. A concomitant relative enrichment of thermally stable amino acids, such as ornithine, glycine, and alanine was noticed and suggests that recrystallization of carbonate tests liberated acidic amino acids from proteinaceous matrices. These unstable compounds were subsequently destroyed.

Amino acid distribution corroborate the fact that the spatial range for microbial degradation of organic matter is dependent on sedimentation rates:

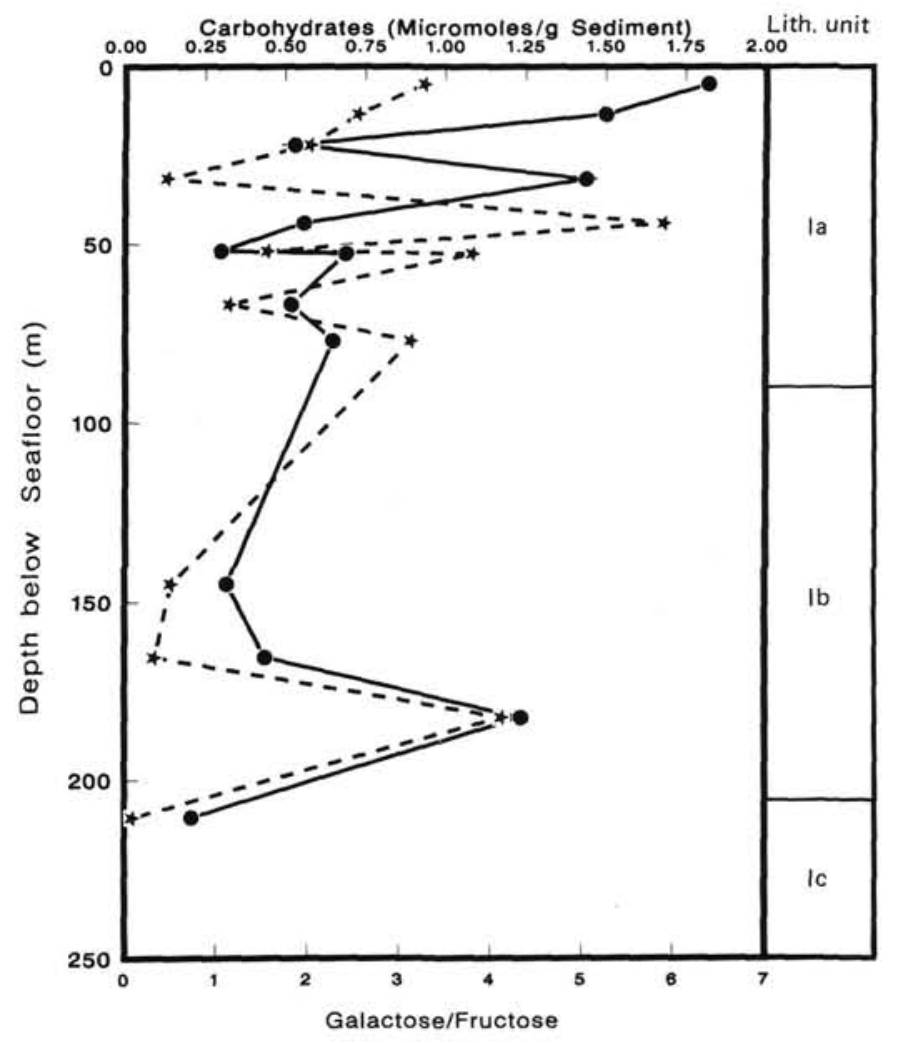

Figure 7. Total carbohydrates in sediment of Site 653 (dots, solid line) in relation to sediment depth, organic carbon content of the sediment, and the ratio of galactose/fructose (stars, broken line). It apppears that the state of degradation decreases as the amount of buried organic matter increases. This observation implies that bacterial degradation does not proceed to completion even though sulfate and labile organic matter is present.

Site 651 has sedimentation rates roughly 10 times those of Site 653 (roughly $40 \mathrm{~cm} / 100 \mathrm{yr}$ vs. $4 \mathrm{~cm} / 1000 \mathrm{yr}$ ), and the zone of amino acid depletion is expanded at Site 651 . Depth within the sediment column does not appear to have an effect on the rate of degradation. Ratios of glutamic acid to $\gamma$-amino butyric acid and of aspartic acid to $\beta$-alanine appear to be useful in tracing ongoing bacterial degradation of sedimentary organic matter even at sediment depths of a few hundred meters. 
Carbohydrate distribution appears to be related to source of organic matter and time. Stable sugars such as fructose increase in their molar contributions relative to more labile sugars such as galactose. The ratio of labile versus stable sugars may be a valuable tool for estimating the state of organic matter degradation in sedimentary sections. It is interesting to note that bacterial decomposition of relatively labile components does not proceed to the point of depletion, even though oxygen donors (sulfate) and labile organic matter (here: galactose) are still present.

\section{ACKNOWLEDGMENTS}

We thank K. A. Kastens, J. Mascle, and F. McCoy for their suggestions on how to improve the manuscript. This study was financially supported by the Deutsche Forschungsgemeinschaft and by a grant from USSAC to K.-C.E. Technical support by the Ocean Drilling Program is gratefully acknowledged.

\section{REFERENCES}

Aizenshtat, Z., Baedecker, M. J., and Kaplan, I. R., 1973. Distribution and diagenesis of organic compounds in JOIDES sediment from Gulf of Mexico and western Atlantic. Geochim. Cosmochim. Acta, 37:1881-1898.

Barker, S. A., Somers, P. J., and Stevenson, J., 1974. Redissolvable ferric-D-fructose and ferric-D-glucose-D-fructose complexes. Carbohydrate Res., 36:331-337.

Bartsch, M., 1987. Laterale und vertikale Verteilung von Zuckern und Aminosäuren im Arabischen Meer-Indikatoren fur Abbauprozesse und Sedimentationsgeschehen. [M.S. thesis] Univ. of Hamburg, p. 126.

Carter, P. W., and Mitterer, R. M., 1978. Amino acid composition of organic matter associated with carbonate and non-cabonate sediments. Geochim. Cosmochim. Acta, 42:1231-1238.

Charley, P. J., Sarkar, B., Stitt, C. F., and Saltman, P., 1963. Chelation of iron by sugars. Biochim. Biophys. Acta, 69:313-321.

Cowie, G. L., and Hedges, J. I., 1984. Carbohydrate sources in a coastal marine environment. Geochim. Cosmochim. Acta, 48:20752087.

Degens, E. T., 1970. Molecular nature of nitrogenous compounds in seawater and recent sediments. In Hood, D. W.(Ed.), Organic Matter in Natural Water. Inst. Mar. Sci., Alaska, 1:77-106.

1976. Molecular mechanisms on carbonate, phosphate and silica deposition in the living cell. Topics in Current Chemistry, 64: $1-112$.

Degens, E. T., and Mopper, K., 1975. Early diagenesis of organic matter in marine soils. Soil Sci., 199:65-72.

1976. Factors controlling the distribution and early diagenesis of organic material in marine sediments. In Riley, J. P., and Chester, R. (Eds.), Chemical Oceanography, 6, London (Academic Press), 75-78.

Emeis, K.-C., Mycke, B., Richnow, H.-H., Spitzy, A., and Degens, E. T., 1987. Organic carbon and nitrogen, sediment composition, and clay mineralogy of DSDP Site 603, Western Atlantic Ocean. In Van Hinte, J. E., Wise, S. W., Jr., et al., Init. Repts. DSDP, 93: Washington (U.S. Govt. Printing Office), 1245-1256.

Gieskes, J. M. and Peretsman, G., 1986. Water-Chemistry Procedures aboard JOIDES Resolution-Some Comments. ODP Tech. Note, 5: $1-46$.

Goossens, H., Irene, W., Rijpstra, C., Duren, R. R., De Leeuw, J. W., and Schenck, P. A., 1986. Bacterial contribution to sedimentary organic matter; a comparative study of lipid moieties in bacteria and recent sediments. In Leythaeuser, D., and Rullkötter, J. (Eds.), Advances in Organic Geochemistry 1985: Oxford (Pergamon Press), 683-696.

Hare, P. E., 1973. Amino acids, amino sugars, and ammonia in sediments from the Cariaco Trench. In Heezen, B. C., and MacGregor, I. D., et al., Init. Repts. DSDP, 20: Washington (U.S. Govt. Printing Office), 941-942.

Hecky, R. E., Mopper, K., and Degens, E. T., 1973. The amino acid and sugar composition of diatom cell-walls. Mar. Biol., 19:323-331.

Hedges, J. I., and Hare, P. E., 1987. Amino acid adsorption by clay minerals in distilled water. Geochim. Cosmochim. Acta, 51:255259.

Henrichs, S. M., and Farrington, J. W., 1987. Early diagenesis of amino acids and organic matter in two coastal marine sediments. Geochim. Cosmochim. Acta, 51:1-15.
Itihara, Y., 1973. Amino acids in the Cenozoic sediments of Japan. Pac. Geol., 6:51-63.

Ittekkot, V., Brockmann, U., Michaelis, W., and Degens, E. T., 1981. Dissolved free and combined carbohydrates during a phytoplancton bloom in the Northern North Sea. Mar. Ecol., 4:299-305.

Kandler, O., 1979. Zellwandstrukturen bei Methan-Bakterien. Naturwissenschaften, 66:95-105.

1981. Archaebakterien und Phylogenie der Organismen. $\mathrm{Na}$ turwissenschaften, 68:183-192.

Kandler, O., and König, H., 1978. Chemical composition of the Peptidoglycan-free cell walls of methanogenic bacteria. Arch. Microbiol., 118:114-152.

Kastens, K. A., Mascle, J., et al., 1987. Proc. ODP, Init. Repts., 107: College Station, TX (Ûcean Drilling Program).

Khan, S. U., and Sowden, F. J., 1971. Thermal stabilities of amino acid components of humic acids under oxydative conditions. Geochim. Cosmochim. Acta, 35:854-858.

King, K., Jr., and Hare, P. E., 1972. Amino acid composition of planktonic foraminifera: A paleobiochemical approach to evolution. Science, 175:1461-1463.

Maita, Y., Montani, S., and Ishii, J., 1982. Early diagenesis of amino acids in Okhotsk Sea sediments. Deep-Sea Res., 29(4A):485-498.

Michaelis, W., Mycke, B., Vogt, B., Schütze, G., and Degens, E. T., 1982. Organic geochemistry of interstitial waters, Sites 474 and 479 , Leg 64. In Curray, J.R., Moore, D.G., et al., Init. Repts. DSDP, 64: Washington (U.S. Govt. Printing Office), 933-937.

Michaelis, W., and Ittekkot, V., 1982. Biogeochemistry of rivers: Field and analytical techniques. In Degens, E. T. (Ed.), Transport of Carbon and Minerals in Major World Rivers, Pt.1. Mitt. Geol. Paläontol. Inst. Univ. Hamburg, 52:69-89.

Michaelis, W., Mycke, B., and Richnow, H.-H., 1986. Organic chemi$\mathrm{cal}$ indicators for reconstructions of Angola Basin sedimentation processes. In Degens, E. T., Meyers, A., and Brassell, S. C. (Eds.), Biogeochemistry of Black Shales. Mitt. Geol. Paläontol. Inst. Univ. Hamburg, SCOPE/UNEP Sonderband, 60:99-113.

Mopper, K., 1977. Sugars and uronic acids in sediments and water from Black Sea and North Sea with emphasis on analytical techniques. Mar. Chem., 5:585-603.

Mopper, K., Michaelis, W., Garrasi, C., and Degens, E. T., 1978. Sugars, amino acids and hydrocarbons in Black Sea sediment from DSDP Leg 42 cores. In Ross, D. A., Neprochnov, Y. P., et al., Init. Repts. DSDP, 42, Pt. 2: Washington (U.S. Govt. Printing Office), 697-705.

Mopper, K., Dawson, R., Liebezeit, G., and Ittekkot, V., 1980. The monosaccharide spectra of natural waters. Mar. Chem., 10:55-66.

Mueller, H., and Bandaranayake, W. M., 1983. An automated method for determination of dissolved organic carbon in seawater using continuous thin-film UV oxidation. Mar. Chem., 12:59-68.

Schroeder, R. A., 1975. Absence of $\beta$-alanine and $\gamma$-aminobutyric acid in cleaned foraminiferial shells: Implications for use as a chemical criterion to indicate removel of non-indigenous amino acid contaminants. Earth Planet. Sci. Lett., 25:274-278.

Seifert, R., and Ittekkot, V., 1985. Dissolved sugars and amino acids associated with "Humic Matter" in the Ems and Elbe rivers. In Degens, E. T., Kempe, S., and Herrera, R. (Eds.), Transport of Carbon and Minerals in Major World Rivers Part 3. Mitt. Geol. Paläontol. Inst. Univ. Hamburg, SCOPE/UNEP Sonderband, 58:413-416.

Spitzy, A., 1982. Amino acids and sugars in deep and shallow groundwater in Hamburg. In Degens, E. T. (Ed.), Transport of Carbon and Minerals in Major World Rivers Part 1. Mitt. Geol. Palaontol. Inst. Univ. Hamburg, SCOPE/UNEP Sonderband, 52:743-748.

Vallentyne, J. R., 1964. Biogeochemistry of organic matter-II Thermal reaction kinetics and transformation products of amino compounds. Geochim. Cosmochim. Acta, 28:157-188.

1969. Pyrolysis of amino acids in Pleistocene Mercenaria shells. Geochim. Cosmochim. Acta, 33:1453-1458.

Weiner, S., Taub, W., and Lowenstam, H. A., 1983. Organic matrix in calcified exoskeletons. In Westbroek, P., and De Jong, E. W. (Eds.), Biomineralization and Biological Metal Accumulation. Dordrecht (D. Reidel), 205-224.

Whelan, J. K., 1977. Amino acids in a surface sediment core of the Atlantic abyssal plain. Geochim. Cosmochim. Acta, 41:803-810.

Date of initial receipt: 26 January 1988

Date of acceptance: 15 September 1988

Ms no. 107B-143 